\title{
Medication Overload: America's Other Drug Problem
}

How the drive to prescribe is harming older adults

\section{April 2019}

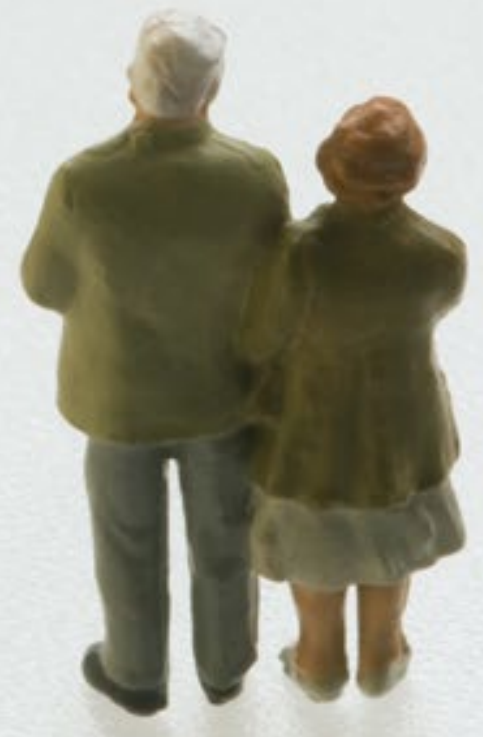




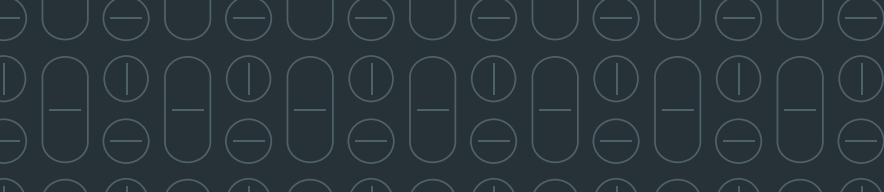

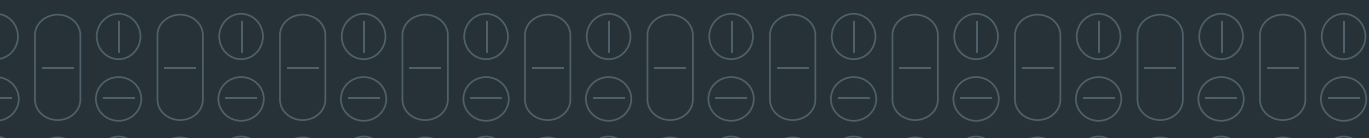
I.

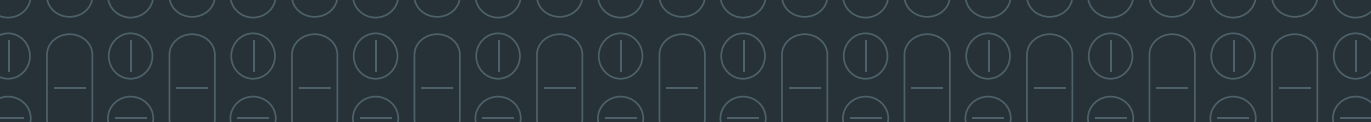

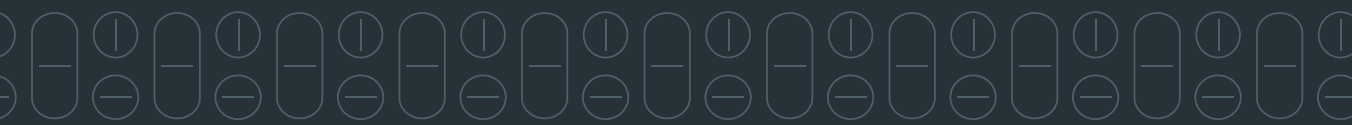
20

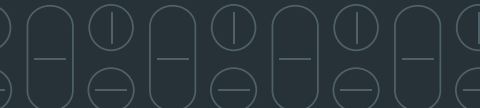

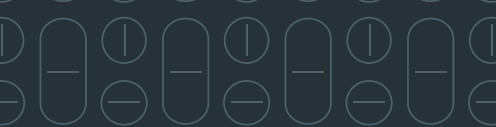

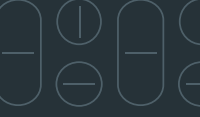
$0 \bigcirc$

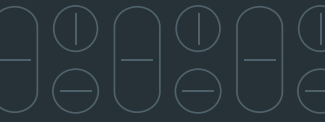

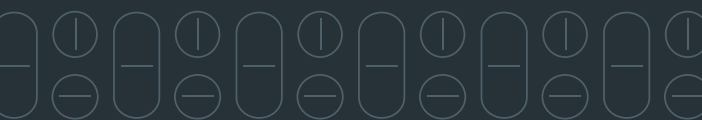

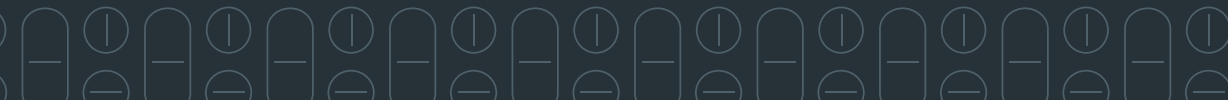
(1) (1) (1) (1) $\Omega \cap \cap \Omega(1$

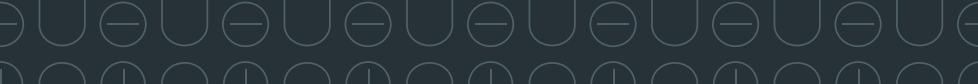

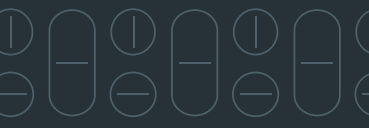

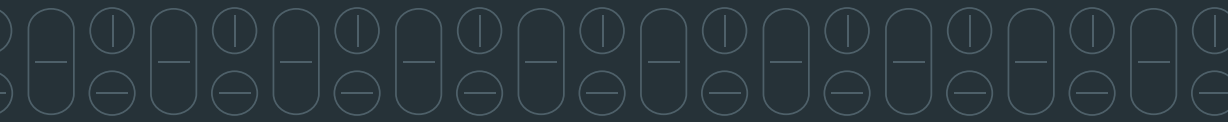

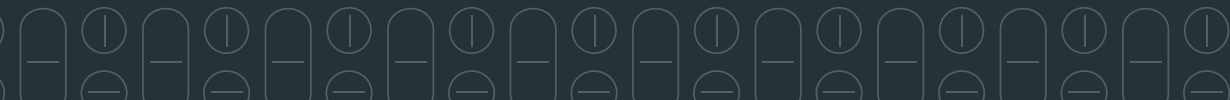

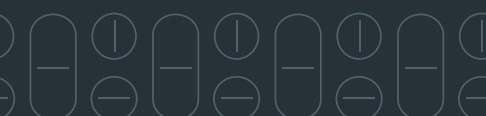

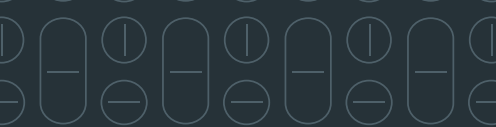

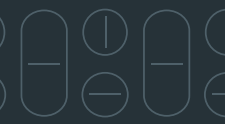
(1)

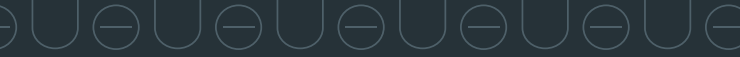

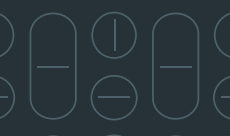
(1) ( 


\section{Medication Overload: America's Other Drug Problem}

How the drive to prescribe is harming older adults

April 2019 


\section{About the Lown Institute}

The Lown Institute is a nonpartisan think tank dedicated to transforming America's high-cost, low-value health system. We conduct research, generate bold ideas, and create a vision for a just and caring system of health that works for all. 


\section{Table of Contents}

\begin{tabular}{l|l} 
PART 1 \\
Medication Overload: \\
Understanding the Scope and Impact
\end{tabular}

(16) Drivers of Medication Overload

(26) Interventions to Address Medication Overload

39 Appendices and References 


\section{PART 1}

\section{Medication Overload: Understanding the Scope and Impact}

(1) $-(1)$

(1)

(1)

$\ominus$

(1) -$]^{\ominus}$

$\ominus$
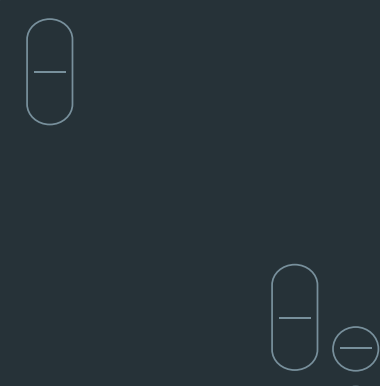

$\theta$

$\ominus$

$\stackrel{\ominus}{\ominus}-$

$\Theta$

$\ominus-\Theta$

$\stackrel{\ominus}{\ominus} \stackrel{(1)}{\ominus}-$ $\ominus$

(1)

$\ominus$

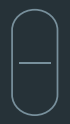

(

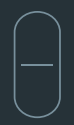

$-(1)$

$\ominus$

$\ominus$

(1)

$\ominus$

-

$\ominus$

$\ominus-\stackrel{1}{\ominus}$

(1) 


\section{Introduction:}

\section{An Epidemic of}

Too Much Medication

Every day, 750 older people living in the United States (age 65 and older) are hospitalized due to serious side effects from one or more medications. ${ }^{1}$ Over the last decade, older people sought medical treatment or visited the emergency room more than 35 million times for adverse drug events, and there were more than 2 million hospital admissions for serious adverse drug events (see Appendix A, p. 40). ${ }^{2}$ Older adults are hospitalized for adverse drug events at a greater rate than the general population is hospitalized for opioids. ${ }^{3}$

In the past decade, prescribing multiple medications to individual patients (called "polypharmacy" in the scientific literature) has reached epidemic proportions.More than four in ten older adults take five or more prescription medications a day, an increase of 300 percent over the past two decades. ${ }^{4}$ Nearly 20 percent take ten drugs or more. When over-thecounter medications and supplements are included, the number of older people taking five or more drugs rises to 67 percent. 5

Medications have improved the lives of individuals around the world, and many patients benefit from taking multiple drugs. Indeed, polypharmacy may be necessary for people who have more than one chronic disease. However, taking multiple medications also greatly increases a person's risk of suffering a serious, sometimes life-threatening side effect. Over the past few decades, medication use in the U.S., especially for older people, has gone far beyond necessary polypharmacy, to the point where millions are overloaded with too many prescriptions and are experiencing significant harm as a result.

\section{Polypharmacy vs. Medication Overload}

\section{Polypharmacy}

A term used in the scientific literature to describe the condition of taking multiple medications. Usually the threshold for polypharmacy is five or more medications, although the cutoff varies because there is not a single agreed upon definition. Polypharmacy can be helpful or harmful, depending on the patient's conditions and the specific medications.

\section{Medication Overload}

The use of multiple medications for which the harm to the patient outweighs the benefit. There is no strict cutoff for when the number of medications becomes harmful, but the greater number of medications a person is taking, the greater their likelihood of experiencing harm, including serious adverse drug events. 
As the number of prescriptions has exploded, so has the frequency of polypharmacy-related harm, what we refer to in this paper as medication overload (see Polypharmacy vs. Medication Overload, p. 03). The epidemic of medication overload is bound to get worse, given the rapid aging of the population and the trend of increasing medication use. Over the next ten years, there will be at least 4.6 million hospitalizations of older Americans and 15 times as many outpatient visits for side effects from medications. Yet the enormity of the problem is invisible to the vast majority of families and patients, most policymakers, and even many health care professionals. And while some clinicians and pharmacists are trying to reduce the burden of medications on their individual patients, no health care professional group, public organization, or government agency to date has formally assumed responsibility for addressing this national problem.

The problem of medication overload has remained hidden largely because the practice of excessive prescribing is deeply embedded in American health care. Like the air we breathe, the forces that lead to medication overload are everywhere and yet difficult to see. No single drug or class of drugs is the culprit, nor is any single disease.

Older people are prescribed drugs in nearly all health care settings, by primary care physicians, specialists, and other health care professionals such as nurse practitioners. In most cases, no single health care professional is assigned responsibility — or has the time, training, and resources - to coordinate patient care, keep track of all the drugs patients have been prescribed, and protect them from medication overload.

Numerous forces and incentives in the U.S. health care system make it easy for clinicians to prescribe medications and difficult to scale back the dosage or deprescribe (stop a prescription). This culture of prescribing is not the fault of any one group. Health care providers, patients, and industry all play a role. There is no magic bullet solution, no single technology, no one policy that can address this problem. A holistic, multi-pronged, and coordinated set of solutions will be necessary to make a meaningful dent in medication overload and the serious side effects that it can cause.

In this report, the Lown Institute calls for the development of a national strategy to address medication overload and help older people avoid its devastating effects on the quality and length of their lives. This report frames medication overload as a critical health care issue, outlining the scope of the problem and the harms that result; it analyzes how the culture, structure, and practices of the health care system both promote medication overload and stand in the way of health care professionals addressing it. Finally, we conclude by outlining some short-term and long-term solutions. A subsequent National Action Plan for Addressing Medication Overload will lay out a national strategy to address the epidemic of prescribing and ensure the safety of millions of older adults who are now at risk of preventable harm and premature death.
Over the last decade, older people sought medical treatment or visited the emergency room more than 35 million times for adverse drug events, and there were more than 2 million hospital admissions for serious adverse drug events.
Numerous forces and incentives in the U.S. health care system make it easy for clinicians to prescribe medications and difficult to scale back the dosage or deprescribe (stop a prescription). 


\section{Polypharmacy can be beneficial or harmful}

Medication overload (harmful polypharmacy) can happen when a patient is prescribed medications that are not necessary, when the side effects from indicated medications outweigh the potential benefit, when a patient is prescribed a medication for longer than needed, and when a patient takes over-the-counter medications or supplements unnecessarily.

\section{Polypharmacy}
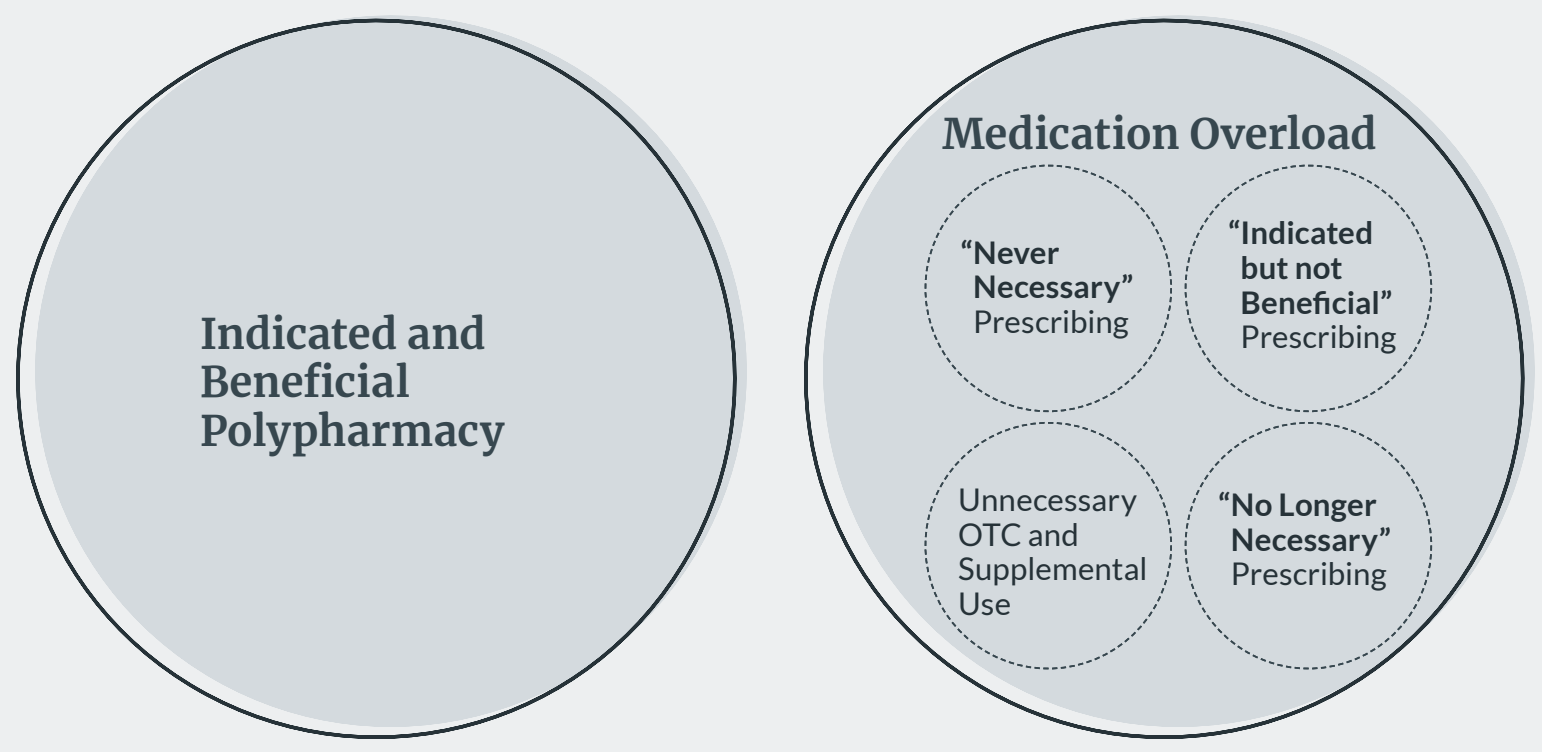


\section{Polypharmacy: A Red Flag for Potential Harm}

In the scientific literature, polypharmacy is most often defined as taking five or more drugs. ${ }^{6}$ This does not mean that every person taking five or more drugs will suffer an adverse drug reaction; some people with multiple chronic conditions require multiple medications to stay healthy. However, the more medications a person takes, the greater their risk of a serious, potentially life-threatening side effect from one or more of these drugs, or interactions between them. ${ }^{2,7,8}$ In this way, the threshold of five or more medications serves as a "risk factor," like high blood pressure, a red flag that a patient is at increased risk of serious harm.

Drug reactions range from relatively mild symptoms such as drowsiness, nausea, or incontinence to more debilitating symptoms such as delirium (and the persistent cognitive changes often associated with it), falls, serious bleeding (including in the brain), or death. When injury occurs as the result of a drug, it is called an Adverse Drug Event (ADE). ADEs include adverse drug reactions, medication errors, allergic reactions, and overdoses.*

While prescriptions are written with the intention of helping patients live better and longer lives, the cumulative effect of multiple drugs can be devastating (see What are the Harms of Medication Overload?, p. 08).

\author{
The threshold of five or \\ more medications serves \\ as a "risk factor", like high \\ blood pressure, a red flag \\ that a patient is at increased \\ risk of serious harm.
}

\section{The risk of an adverse drug event increases by $7-10 \%$ with each medication.}

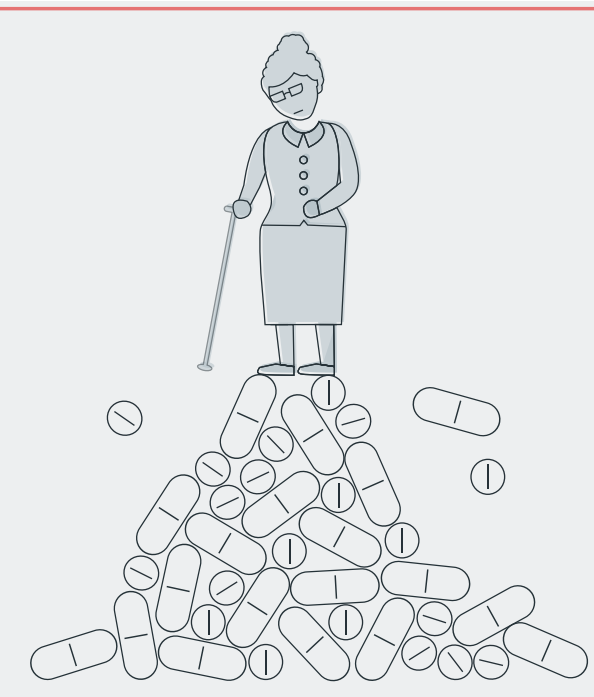

* An adverse drug reaction is defined as a negative reaction from use of a medication as prescribed. Adverse drug events include adverse drug reactions, but also include negative effects from non-regular use of a medication, such as medication errors and overdoses. 
ADEs are one of the most common causes of medical harm, especially for older people. In 2014, the rate of Emergency Department visits for ADEs among older Americans was nearly 10 per 1000 individuals. ${ }^{15}$ At a minimum, about 200,000 older adults were hospitalized that year because of an adverse drug event, and as many as 878,000 hospital stays for older adults included some kind of adverse drug event present on admission..$^{1,15}$ People over 65 make up only about 14 percent of the population, but they account for 56 percent of the country's hospitalizations for adverse drug events. ${ }^{15}$

Additionally, many older adults seek medical care for ADEs at the offices of their primary care doctors, outpatient clinics, or emergency departments but are never hospitalized. Older adults taking five or more medications are at least 88 percent more likely to seek outpatient care for an adverse drug event compared to those taking just one or two. ${ }^{2}$

Taking into account the growth in the older population and increases in rates of ADEs over the last decade, we estimate that there have been nearly 5 million outpatient visits for ADEs for older adults in 2018, and 280,000 hospitalizations, at a cost of $\$ 3.8$ billion (see Appendix A, p. 40). At the same time, less than half of people experiencing an $\mathrm{ADE}$ recognize it and seek medical treatment, meaning that about ten million older people in the U.S. - about one in five - suffer from an adverse drug event each year. ${ }^{2}$

The potential ill effects of excessive prescribing go beyond ADEs and hospitalizations. People can be confused and overwhelmed by having to keep track of numerous medications - when they should be taken, how they should be taken, what they're for. Despite the best efforts of community pharmacists, family, and in some cases, long-term care facility staff, the mental effort of managing this "pill burden" is too much for many people, making it difficult for them to maintain their medication regimen.
Older adults taking five or more medications are at least $\mathbf{8 8}$ percent more likely to seek outpatient care for an adverse drug event compared to those taking just one or two.
In 2018, there were more than
$\mathbf{2 8 0 , 0 0 0}$ hospitalizations
of older people in the U.S.
because of an adverse drug
event, at a cost of \$3.8 billion.

“Sometimes my patients' pills don't fit in one pill container, so they have to buy two. Taking so many medications, it gets to them."

- Anonymous clinician participating in a focus group 


\section{What are the Harms of Medication Overload?}

Each additional medication prescribed increases the risk of harmful side effects, such as delirium, falls, and other adverse events that can be life-threatening.

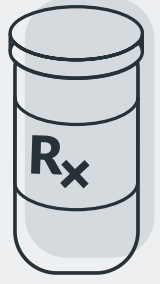

\section{Adverse Drug Events}

For each additional drug in a patient's medication regimen, the risk of adverse drug events increases by 7 to 10 percent. ${ }^{7,8,9}$

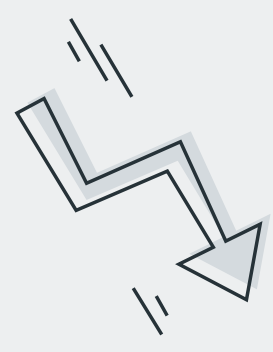

\section{Falls}

For older adults, taking 4 or more drugs is associated with an 18 percent greater risk of falls. Taking 10 or more drugs is associated with a 50 percent higher risk of falls. ${ }^{12,13}$

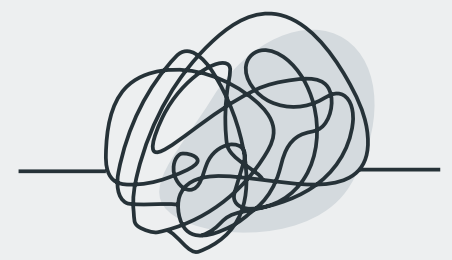

\section{Delirium}

Older patients taking 6 or more drugs in the hospital are more than twice as likely to experience delirium compared to patients taking fewer drugs. ${ }^{10}$ Older people taking more than 10 drugs are nearly 2.5 times more likely than those taking fewer than 5 drugs to experience impaired cognition. ${ }^{11}$

\section{Mortality}

For older adults, taking 6 to 9 medications is associated with a 59 percent greater chance of death compared to taking no medications. Taking 10 or more medications is associated with a 96 percent greater chance of death. ${ }^{14}$ 
Medication adherence - i.e., taking medications as prescribed decreases as the number of prescriptions increases: about 80 percent of patients on one medication take them as prescribed, compared to just 51 percent of patients on four or more medications. ${ }^{22}$ Ironically, when patients are overburdened by prescriptions, they may neglect critically important drugs, which can lead to a deterioration in health and a decline in quality of life. ${ }^{23}$

Patients taking multiple drugs are also burdened by the financial cost. Although most older people in the U.S. have prescription drug coverage under Medicare Part D, many still have trouble with out-of-pocket medication expenses; in 2015, more than 40 percent of adults over 50 indicated in a survey that they were concerned about being able to afford their medications. ${ }^{24}$ High costs can lead to non-adherence and anxiety. People may ration their medications, which in turn can create even more medical problems. ${ }^{25}$

\section{A week's worth of medication for a 92-year old patient, before and after deprescribing.}

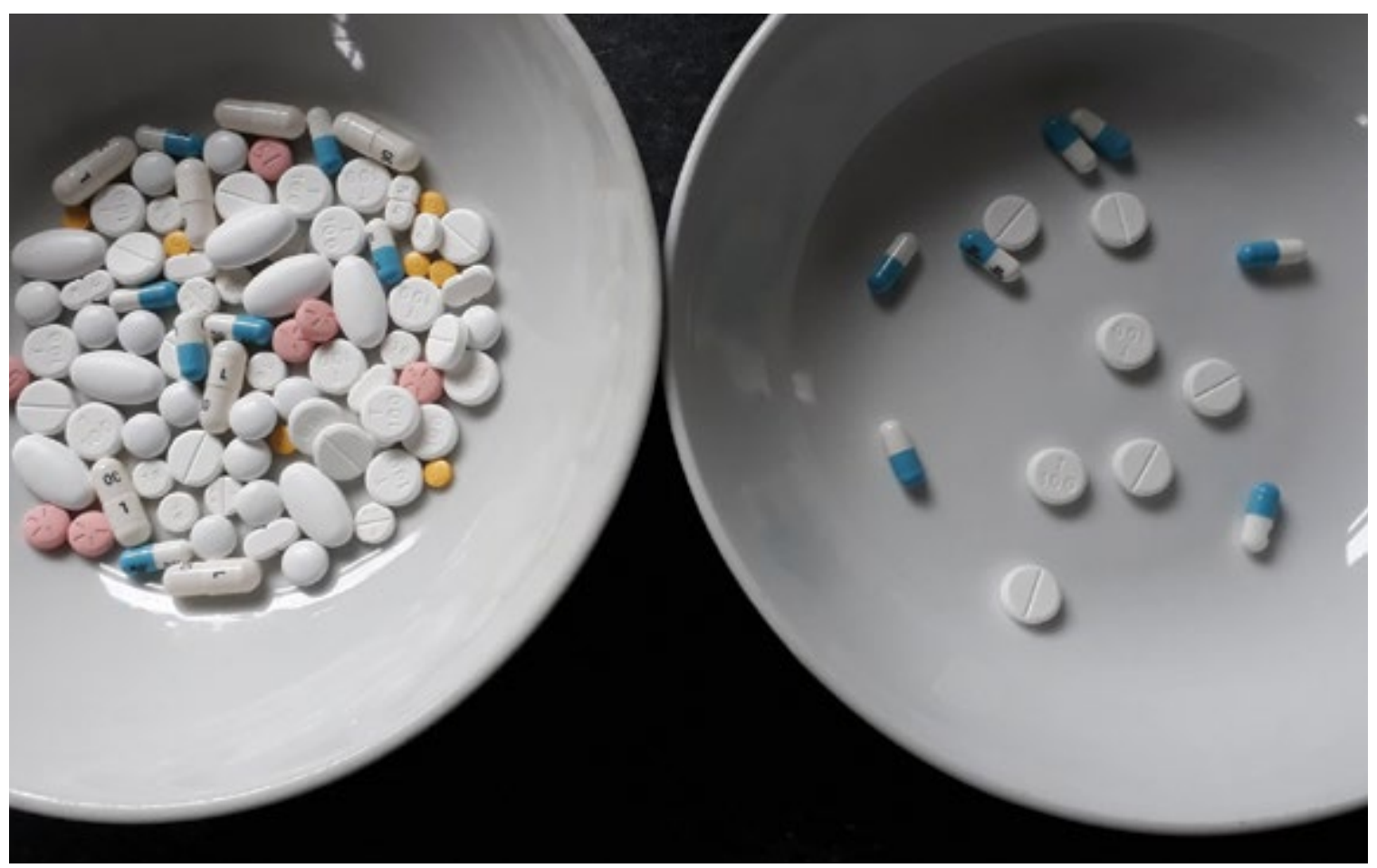

Photo reprinted with permission from Dr. David Alldred 


\section{Medications Most Likely to Cause Harm}

\section{Three classes of drugs} contribute to 60 percent of emergency room visits for adverse drug reactions among older adults. ${ }^{15}$

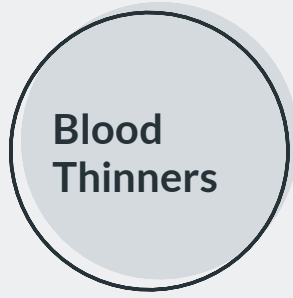

Blood thinners such as aspirin or warfarin can cause severe bleeding, which can be lifethreatening.

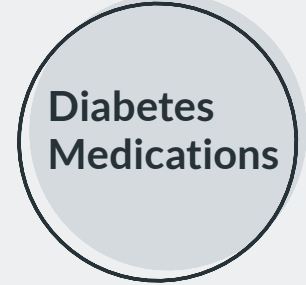

Diabetes medications such as insulin or gliclazide can cause low blood sugar in older adults, increasing risk for falls, fractures, confusion, seizures, and hospitalization.

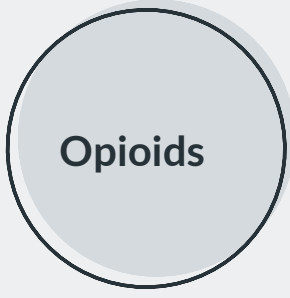

Opioids prescribed to treat pain in older adults can be habit-forming and can lead to sedation, falls, cognitive impairment, and motor vehicle accidents.

Other classes of drugs have been shown to increase the potential for harmful side effects.

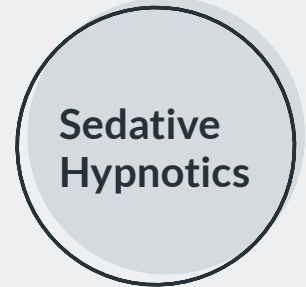

Sedative hypnotics such as benzodiazepines (anti-anxiety medications) and many sleep

medications, as well as antidepressants, increase the risk of falls, fracture, cognitive impairment, and other adverse effects. ${ }^{18,19}$

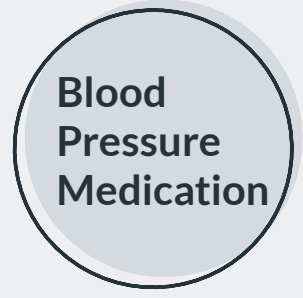

Blood pressure medications, especially when used at high doses or in combination, can lower blood pressure too much and lead to falls, cognitive impairment, and other adverse effects. ${ }^{16}$

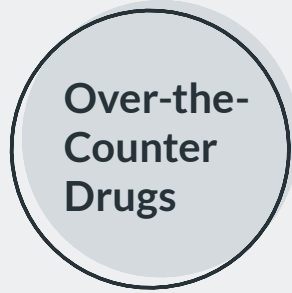

Many commonly used over-the-counter drugs, including bladder medications and antihistamines have anticholinergic properties. These medications can cause hallucinations, confusion, tachycardia, dry eyes, and other adverse effects, which become more pronounced at higher doses and in combination with each other. ${ }^{20,21}$

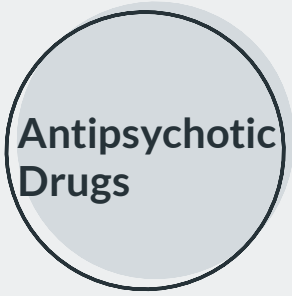

Antipsychotic drugs can lead to delirium, cognitive impairment, drug-induced movement disorders, and metabolic disturbances. ${ }^{17}$ 

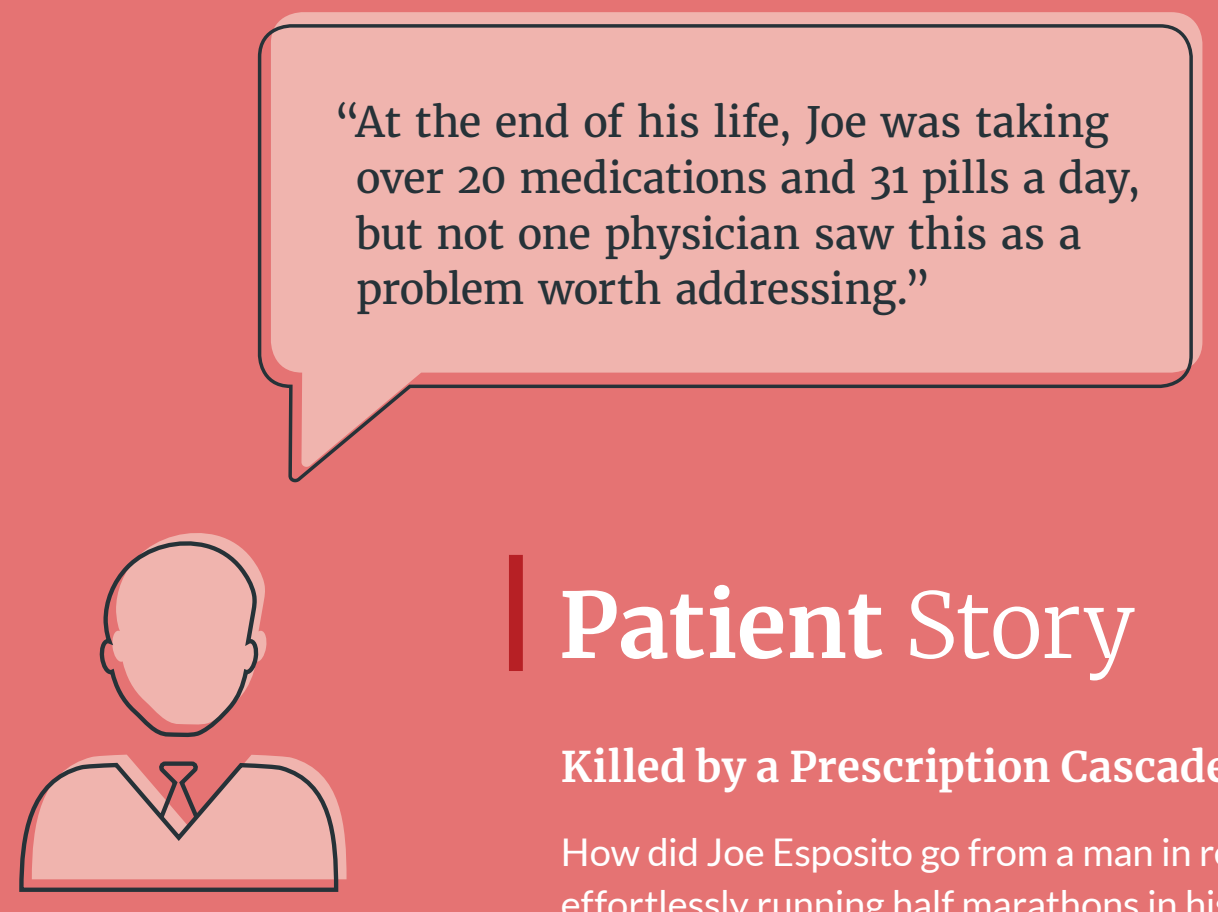

\section{Killed by a Prescription Cascade}

How did Joe Esposito go from a man in remarkable health, effortlessly running half marathons in his 50 s, to being debilitated and incapacitated, struggling at death's door, in just a few years? When Joe sought treatment for his mild to moderate Crohn's disease, his list of medications cascaded from one to six to twenty, as each new medication brought on a new side effect.

The steroids he was prescribed to treat Crohn's led to bone loss and anal fistulas. He was given antibiotics for the fistulas, which caused peripheral neuropathy in his feet. He couldn't sleep from the pain so he was prescribed benzodiazepines and Ambien for sleep, Lyrica for the nerve damage, and Tramadol for the pain. Several of the drugs gave him severe diarrhea. To treat his diarrhea, he was given opium drops, and other medications. Additional drugs weakened his kidneys, which in turn raised his blood pressure, so he was put on four blood pressure medications. And an experimental anti-inflammatory drug led to Joe contracting pericardial tuberculosis, which almost killed him.

At the end of his life, Joe was taking over 20 medications and 31 pills a day, but not one physician saw this as a problem worth addressing or even considered that his symptoms were caused by the drugs and not the Crohn's disease. 


\section{Prevalence of polypharmacy and medication overload: The "new normal"}

The 21st century has already become the Era of the Prescription. In 2000, half of adults in the U.S. were taking at least one prescription drug; by 2012, that number had grown to nearly 60 percent. ${ }^{26}$ Not only are more people taking prescription drugs, they are taking a greater number of drugs. From 2000 to 2012, the proportion of adults in the U.S. taking five or more drugs nearly doubled, from 8.2 percent to 15 percent. ${ }^{26}$

Prescription drug use has become the "new normal" for people of all ages, but especially older people. About 90 percent of people in the U.S. over age 65 take at least one drug; 42 percent take five or more drugs; and at least 18 percent take ten or more drugs per month. ${ }^{4,27}$

From 1994 to 2014, the proportion of older adults taking five or more drugs tripled, from 13.8 percent to 42.4 percent. ${ }^{4}$ Including over-thecounter medications and supplements, 67 percent of older people regularly take five or more drugs. ${ }^{5}$

\section{Groups with higher risk of medication overload}

- Long-term care residents: The rate of polypharmacy is 40 to 50 percent higher for residents in long-term care facilities, compared to older adults living in the community. ${ }^{28}$

- Cancer patients: As many as 84 percent of older cancer patients are taking five or more medications. ${ }^{29,30}$

- Low-income seniors: People who are dual-eligible for Medicare and Medicaid have a 25 percent higher rate of chronic conditions, increasing their risk for medication overload. ${ }^{31}$ 


\section{The Increasing Prevalence of Polypharmacy}

From 1994 to 2014, the proportion of older adults taking five or more drugs tripled, from 13.8 percent to 42.4 percent.

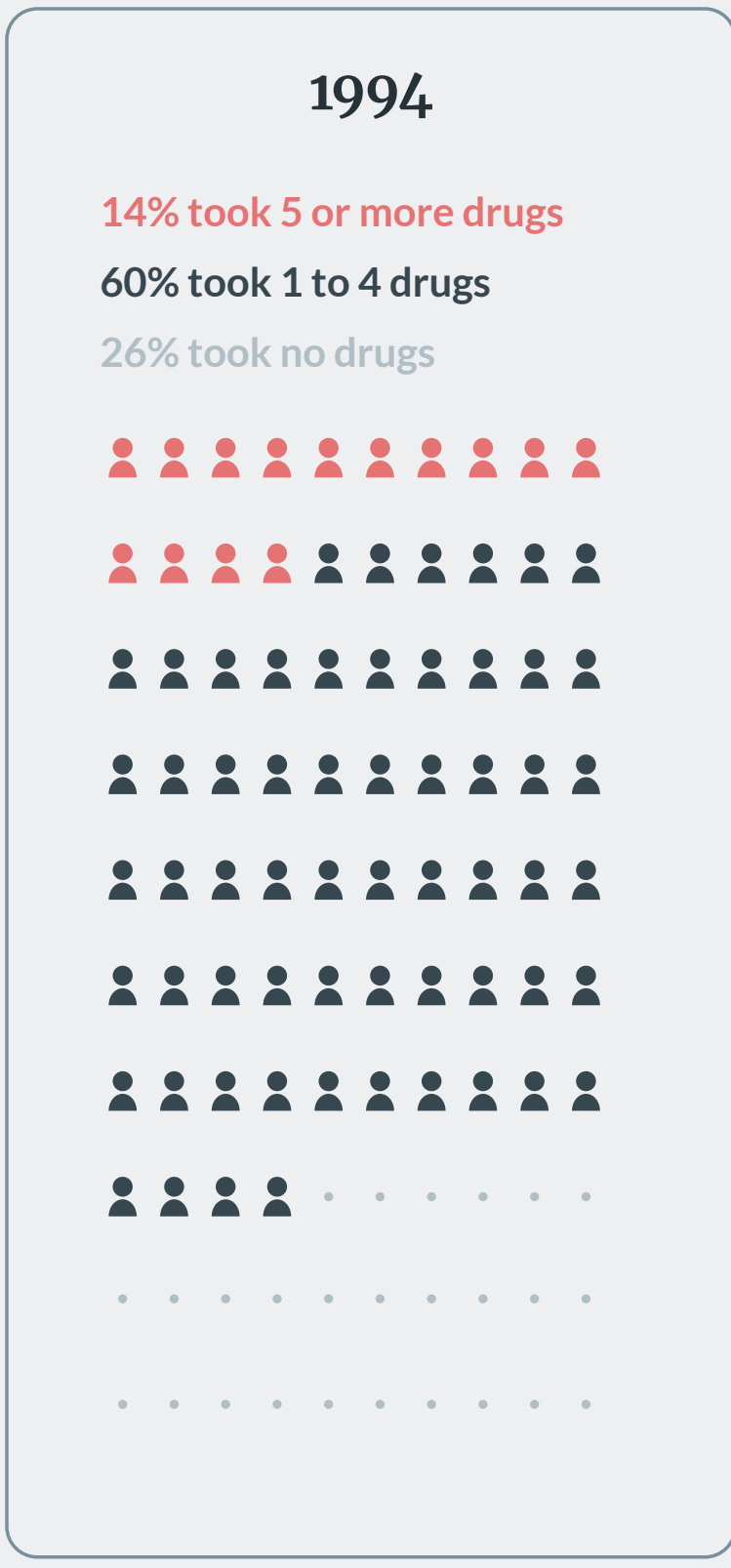

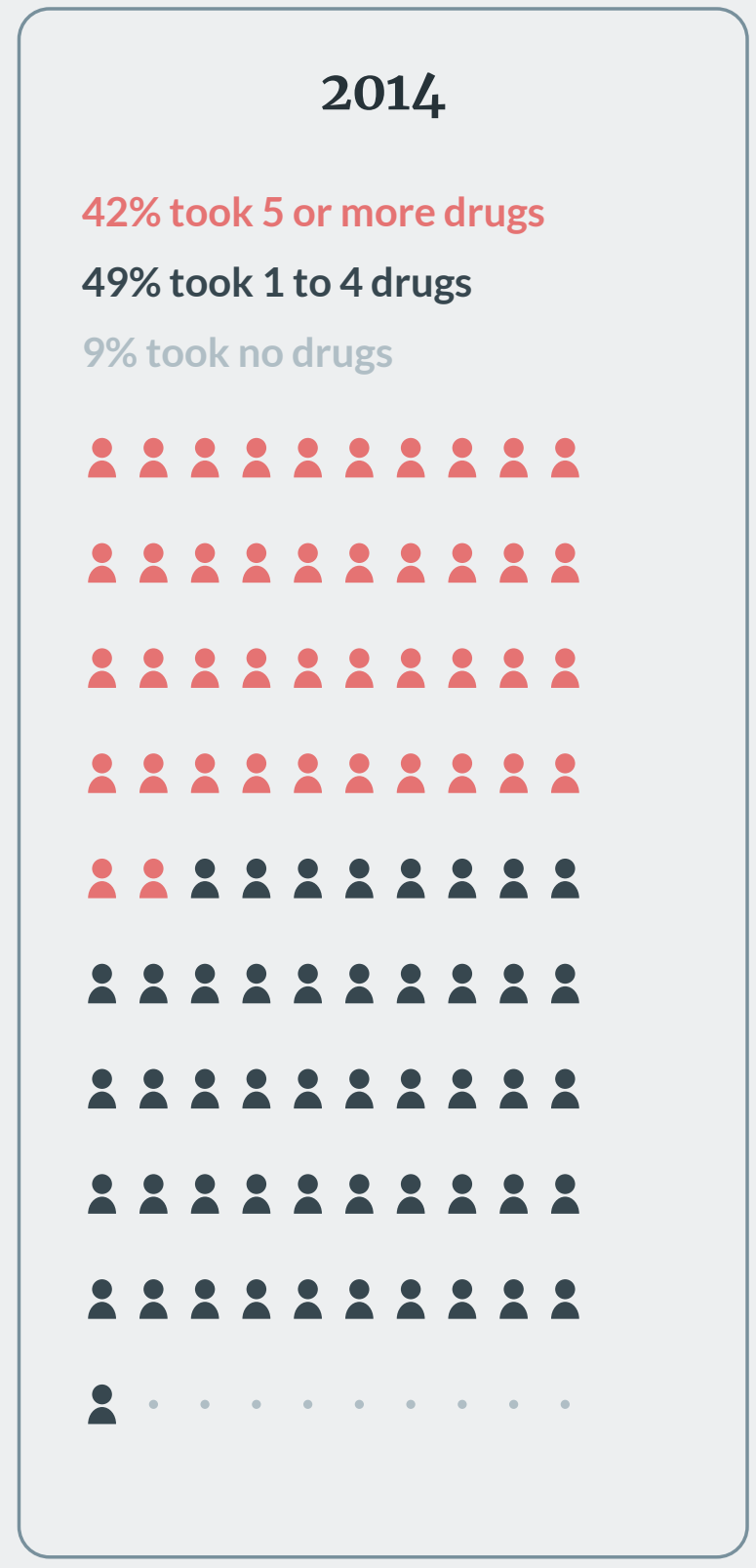




\section{The Rising Tide: Projections of Future Prevalence and Harm From Medication Overload}

Without swift action to reduce medication overload, we face a severe public health crisis in the near future. The rates of prescription drug use and adverse drug events have both increased dramatically over the past decade. Additionally, the U.S. population is rapidly aging, putting at least 20 million more people at risk of medication overload over the next decade. ${ }^{32}$

If nothing is done, adverse drug events will be responsible for at least 4.6 million hospitalizations of older people in the United States over the next decade. These hospitalizations will cost taxpayers, patients and families an estimated $\$ 62$ billion, and lead to nearly 150,000 premature deaths. Additionally, we can expect 74 million outpatient visits as a result of older adults experiencing adverse drug events (see Appendix A, p. 40).

The aging of America is inevitable, but we can lessen the potential harms of ADEs by reducing medication overload. If we could reduce the rate of ADEs by 50 percent, we would prevent about 2.3 million hospitalizations, saving 74,000 lives and \$30 billion, and reduce the number of outpatient visits for ADEs by 37 million between 2020 and 2030 .

What if we could reduce $\mathrm{ADE}$ by $50 \%$ ?

If we could reduce the rate of ADEs

by 50 percent, we would prevent about 2.3 million hospitalizations, saving 74,000 lives and $\$ 30$ billion, and reduce the number of outpatient visits for ADEs by 37 million between 2020 and 2030.

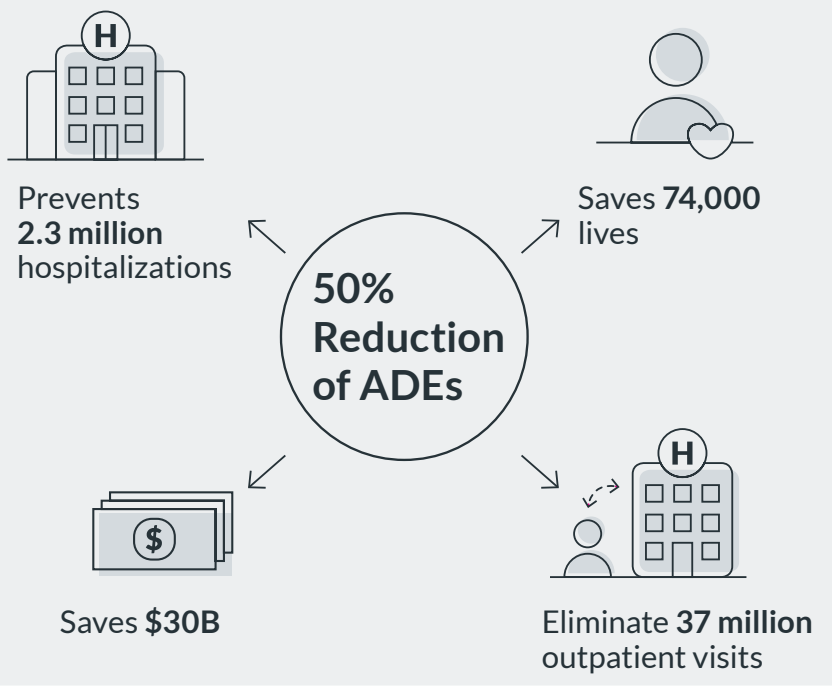




\section{Patient Journey}

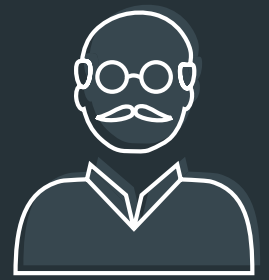

Meet Gary. He lives with his wife in Akron, Ohio.

He's a retired teacher and loves fishing.

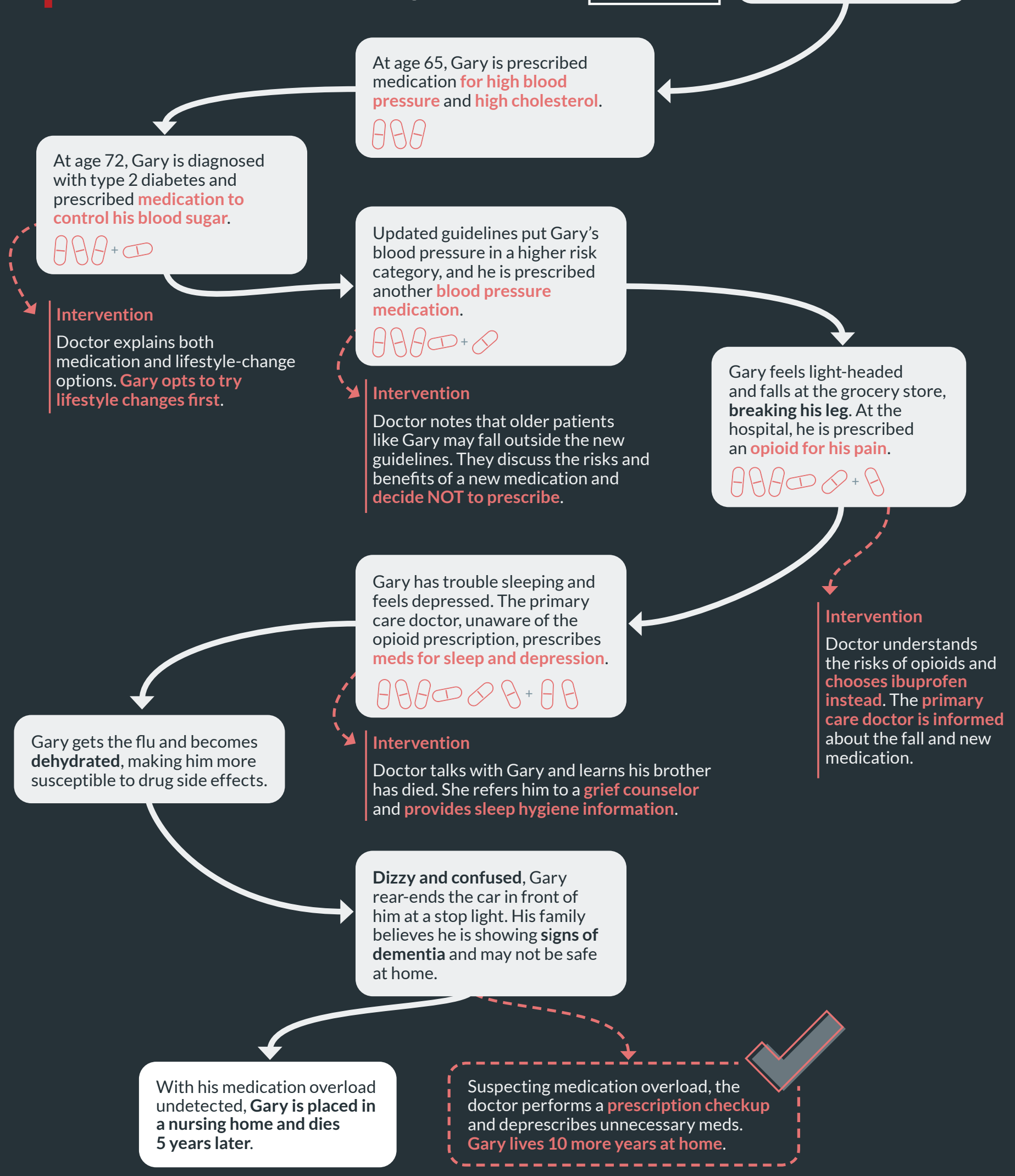

(15)| Medication Overload: America's Other Drug Problem | Lown Institute 


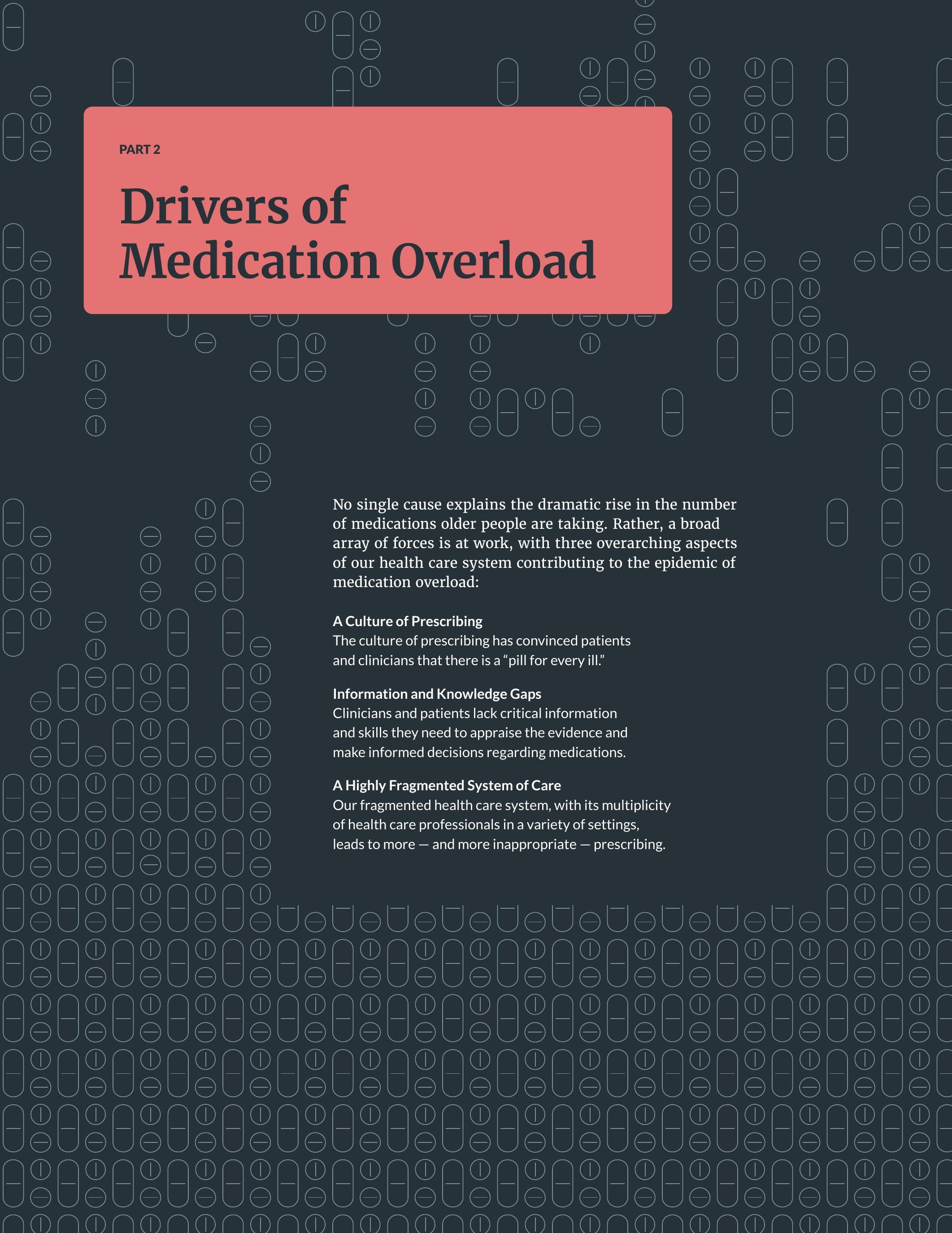




\section{Culture of Prescribing}

Twenty years of advertisements linking prescription medications to happiness and health, the increased medicalization of normal human aging and experiences, the hurried pace of medical care, and a desire on the part of both health care professionals and patients and their families to "do something" have together fostered a shared expectation that there is a "pill for every ill." Multiple factors shape this culture of prescribing and it, in turn, influences the way clinicians and patients think about drugs.

Prescribing is kind
Studies have found that both clinicians and patients think of prescribing medications as "kind." Getting a prescription validates patients. At the same time, health care professionals feel gratified they have done something to help..$^{33}$

Prescribing is faster and easier than addressing lifestyle factors that contribute to chronic illness, such as lack of exercise, smoking, and alcohol use. ${ }^{34}$ Health care professionals may not feel they have the time to discuss lifestyle changes with patients (who may not be open to these changes), or that they are adequately reimbursed to do so. In many cases, clinicians turn to (or patients expect) medications as a "quick fix" for illness or the symptoms of aging, without paying attention to, or being aware of, the potential for harm associated with taking too many. ${ }^{35,36}$

Marketing also contributes to the culture of prescribing. The U.S. is one of only two countries that allows direct-to-consumer pharmaceutical advertising that includes product claims. Drug makers spent $\$ 6$ billion on advertising to patients in 2016, a more than four-fold increase since the late 1990 s $^{37}$ These advertisements, which bombard consumers, heavily promote the benefits of medications while using long, fast-spoken (or small-print) descriptions of harms. In one study of drug advertisements aired in 2015 and 2016, not a single one included a quantitative presentation of the risks..$^{38}$ This lack of context leads viewers to overestimate the benefits of drugs while underestimating the potential harms. ${ }^{39}$

Direct-to-consumer advertisements are also used to redefine normal, everyday symptoms as medical conditions that require pharmaceutical treatment. Known as medicalization, this process contributes to the culture of prescribing. ${ }^{40-42}$ Over the past few decades, drugs have become the first line of treatment for common conditions such as sleeplessness, back pain, and acid reflux, replacing effective non-pharmacological therapies. For recently identified "conditions," medicalization is often the prelude to extensive marketing campaigns for prescription drugs to treat the new "disorders." ${ }^{42}$ Medicalization essentially creates the "ill for every pill." 


\section{Marketing to doctors}

The drug industry also markets heavily to health care professionals, spending more than $\$ 20$ billion a year on face-to-face promotion, free samples, promotional meetings, continuing medical education, and other marketing activities. ${ }^{37,43}$ While the industry is not allowed to market drugs for unapproved conditions, companies often fund studies designed to prove their efficacy for common ailments. ${ }^{44}$ Subsequently published abstracts and papers act as a marketing blitz, telling prescribers that medications are safer, more effective, and appropriate for a larger population of patients than they actually are..$^{45-47}$

The trend of increasing medication use among older adults has contributed to its normalization. As more and more older people take multiple medications, younger people come to expect that they, too, will need numerous drugs when they grow older. Thus, we are "primed" to take multiple medications and are less likely to question our clinician when offered a prescription. ${ }^{48,49}$

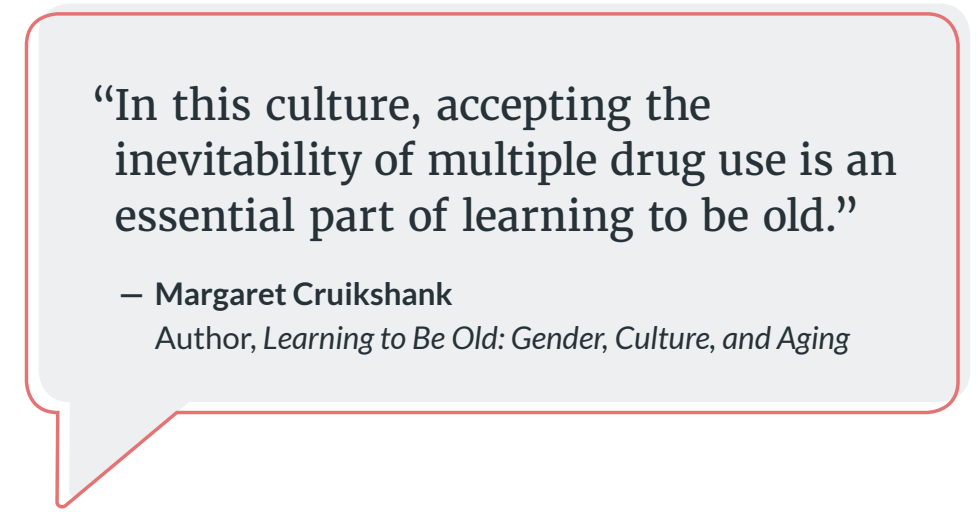

"Starting a drug is like the thrill of marriage and stopping it is like the agony of a divorce."

- Doug Danforth

Pharmacist, Vancouver, British Columbia 


\section{Information and Knowledge Gaps}

Clinicians and patients lack critical information and skills they need to appraise the evidence and make informed decisions regarding medications. ${ }^{50}$ From medical school to residency to continuing medical education (CME), nowhere is learning about the dangers of excessive prescribing a mandated, formal part of the curriculum for physicians. The same is true for nurses, physician assistants, and other health professionals.

\section{Lack of training}

\section{Research gaps}

During the years that physicians, nurses, and pharmacists spend in training, they are rarely taught how to proactively monitor for and avoid medication overload, or how to pause or stop medications. Students and trainees also get scant training in how to adjust treatment for older adults - for example, using lower doses or watching for side effects that can masquerade as disease symptoms..$^{51,52}$ As a result, clinicians often feel unprepared or unqualified to discontinue medications for their patients. Trainees who wish to prescribe less may feel pressure to follow the practice patterns at their institutions set by more senior clinicians, even when these are not based on evidence. ${ }^{53}$

Although the body of research on medication overload is growing fast, many gaps in our knowledge remain (see Gaps in the research: Unanswered questions, p. 32). There is scant information about interactions between drugs in older patients. For health care professionals who are aware of the dangers of medication overload, and who would like to reduce the number of drugs their patients are taking, few guidelines are available for how to do so safely. ${ }^{54}$
"If I lower my patient's blood pressure [to the point where] they fall and break their hip, I still get paid. But if their blood pressure is above the target, I fail the guideline."

- Andy Lazris

Primary care doctor, Columbia, MD 


\section{Inadequate clinical guidelines}

Doctors and other prescribers generally turn to clinical guidelines for guidance in treatment decisions. These guidelines, which are created by physician professional societies, are based on studies of selected patients, who are often younger, healthier, and have fewer chronic illnesses than the patients who will end up being treated. Thus, guidelines routinely do a poor job articulating the risks of medication-related interventions in patient groups (e.g., older adults) not well-represented in clinical studies. ${ }^{54}$

Guidelines often recommend prescribing medications as the first line of treatment and "stepping up" drug regimens when the first effort does not succeed. This often involves higher doses and/or additional medications without consideration of the patient's age, health, disease trajectory, or overall drug burden. ${ }^{54,55,35}$ Guidelines rarely offer advice about lowering doses for older patients ("stepping down" therapy), considering the total number of drugs a patient is on, or considering stopping a medication.

Health care professionals often feel compelled to follow guidelines, even when they know the guidelines may not be entirely appropriate for the individual patient. ${ }^{53}$ Insurers use clinical measurements laid out in guidelines, such as optimal blood pressure readings or cholesterol levels, to set performance standards for physicians and other prescribers. These performance standards are linked to payment and can push health care professionals to prescribe additional drugs, or higher doses to achieve the clinical goal - even when that goal runs counter to that patient's quality of life and even safety. For example, following blood pressure guidelines too diligently can lead to multiple prescriptions that can make older people dizzy and prone to falls (see Clinician story: Hitting the target but missing the mark, p. 21).

The pharmaceutical industry contributes to the lack of reliable information on medications by influencing clinical drug trials (studies involving human subjects). The majority of clinical research is paid for by drug companies, which often design the trials themselves to increase the potential for positive outcomes. Companies also hire medical writers to spin results in ways that highlight the benefits and downplay potential side effects. ${ }^{57-59}$ Industry money plays a role in clinical guidelines as well; many of the professional societies that publish guidelines rely heavily on industry funding, giving them less incentive to promote a culture of deprescribing. ${ }^{60,61}$ 

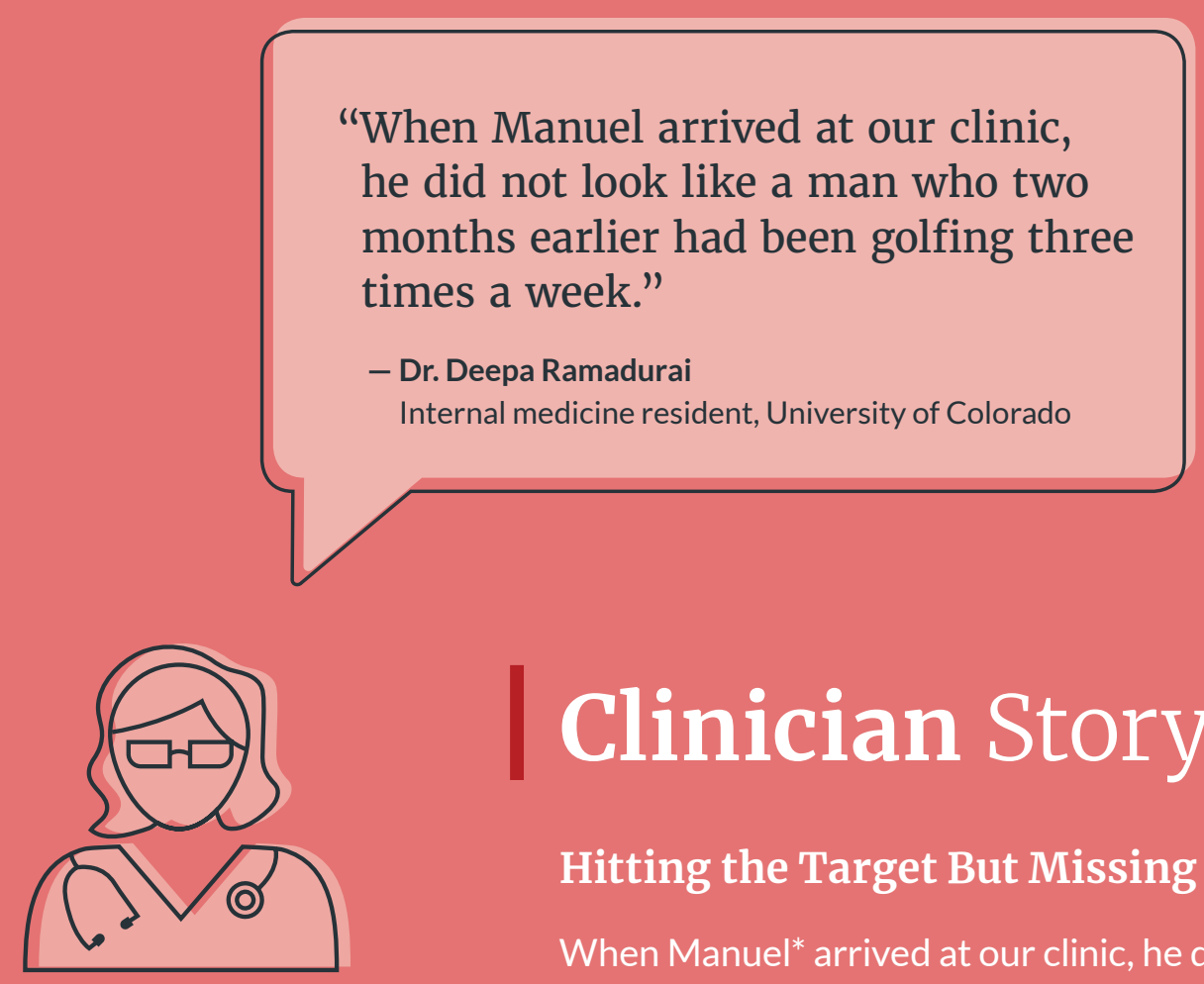

| Clinician Story

\section{Hitting the Target But Missing the Mark}

When Manuel* arrived at our clinic, he did not look like a man who two months earlier had been golfing three times a week and was able to take care of his young grandchildren. He said he was fatigued all the time and often dizzy.

After looking at Manuel's medical record and hearing his story, I had a hunch that polypharmacy was the cause of his symptoms. Considering his age and increased risk factors for heart attack and stroke, it was possible that his previous doctor had increased his blood pressure medications, which can cause lethargy and dizziness.

Despite the potential adverse side effects, older patients are regularly given these medications because physician guidelines recommend more aggressive treatment of high blood pressure for all patients, no matter their age or other conditions.

Sure enough, Manuel's previous doctor had added more medications to his regimen a few months ago to try to lower his blood pressure. He was on four different blood pressure medications - a beta blocker, a vasodilator, a calcium channel blocker, and an angiotensin receptor blocker.

We created a plan based on Manuel's risk profile to gradually taper and eventually discontinue two of the hypertension drugs he was on. Once he stopped taking these drugs, his symptoms of lightheadedness and fatigue stopped completely. His blood pressure also remained stable over the following year. 


\section{Fragmented System of Care}

Lastly, our fragmented health care system, with its multiplicity of health

care professionals in a variety of settings, leads to more - and more

inappropriate - prescribing. Too often, no single clinician or team is

coordinating care and keeping track of all of a patient's prescriptions.

Moreover, individual clinicians often have inadequate time to sort

through medications and remove those that are burdening the patient.

Lack of teamwork

\section{Care transitions}

With health care delivery in the U.S. increasingly specialized, primary care clinicians struggle to serve as the single provider keeping watch over the whole patient. ${ }^{62}$ Rather, each specialist treats each condition a patient has separately, often without knowing or considering what other conditions the patient has or what medications they are already taking. This problem particularly affects older adults, who are more likely to have multiple chronic illnesses and see multiple clinicians..$^{63,64}$

Inappropriate prescribing is made even worse during transitions to and from hospitals, rehabilitation centers, or nursing homes. ${ }^{35}$ In the hospital, patients are often given drugs to treat an acute, temporary condition. Even if these prescriptions are meant to be short-term, they are frequently continued after patients are released from the hospital. ${ }^{65}$

During transitions to residential care settings (e.g., nursing homes), understaffing, lack of familiarity with the patient's medical history, and lack of coordination among clinicians may lead to medication increases. For older adults with cognitive issues, the transition from hospital to long-term care can be destabilizing and upsetting, prompting the use of antipsychotics - one of the most commonly misused class of drugs in nursing homes - to calm the resident. ${ }^{55}$ Despite campaigns to improve nursing home care, such as the CMS National Partnership to Improve Dementia Care in Nursing Homes (see U.S. public policies related to medication overload, p. 34), these drugs continue to be used to sedate patients who are troublesome or disruptive. ${ }^{66}$ 
Poor EMR design

\section{Prescribing cascade}

The lack of communication among health care professionals is exacerbated by inadequate and poorly designed technology. Electronic medical records (EMRs) have been in place in the health care system for several decades but are still frustratingly clunky and difficult to use for clinical purposes (as opposed to billing purposes, for which they were designed). EMRs lack "interoperability" - i.e., different EMR systems don't communicate with each other - making it difficult for clinicians to see what medications others have prescribed for their patients, and for what conditions. ${ }^{67,68}$

As a consequence of specialization and fragmentation, many patients have no single health care provider who knows all of the drugs the patient is on, or why they were prescribed. This makes it difficult for any clinician to take into account the cumulative effect of multiple prescriptions and the potential harm that adding one more may cause. Often, clinicians prescribe drugs to treat what appears to be a new condition or the exacerbation of an existing one, when in reality they are treating a side effect of a drug. When the newest drug causes a new side effect, another clinician may write yet another prescription, causing what's known as a "prescribing cascade," which can be more dangerous than the disease itself (see Patient story: Killed by a prescribing cascade, p. 11).
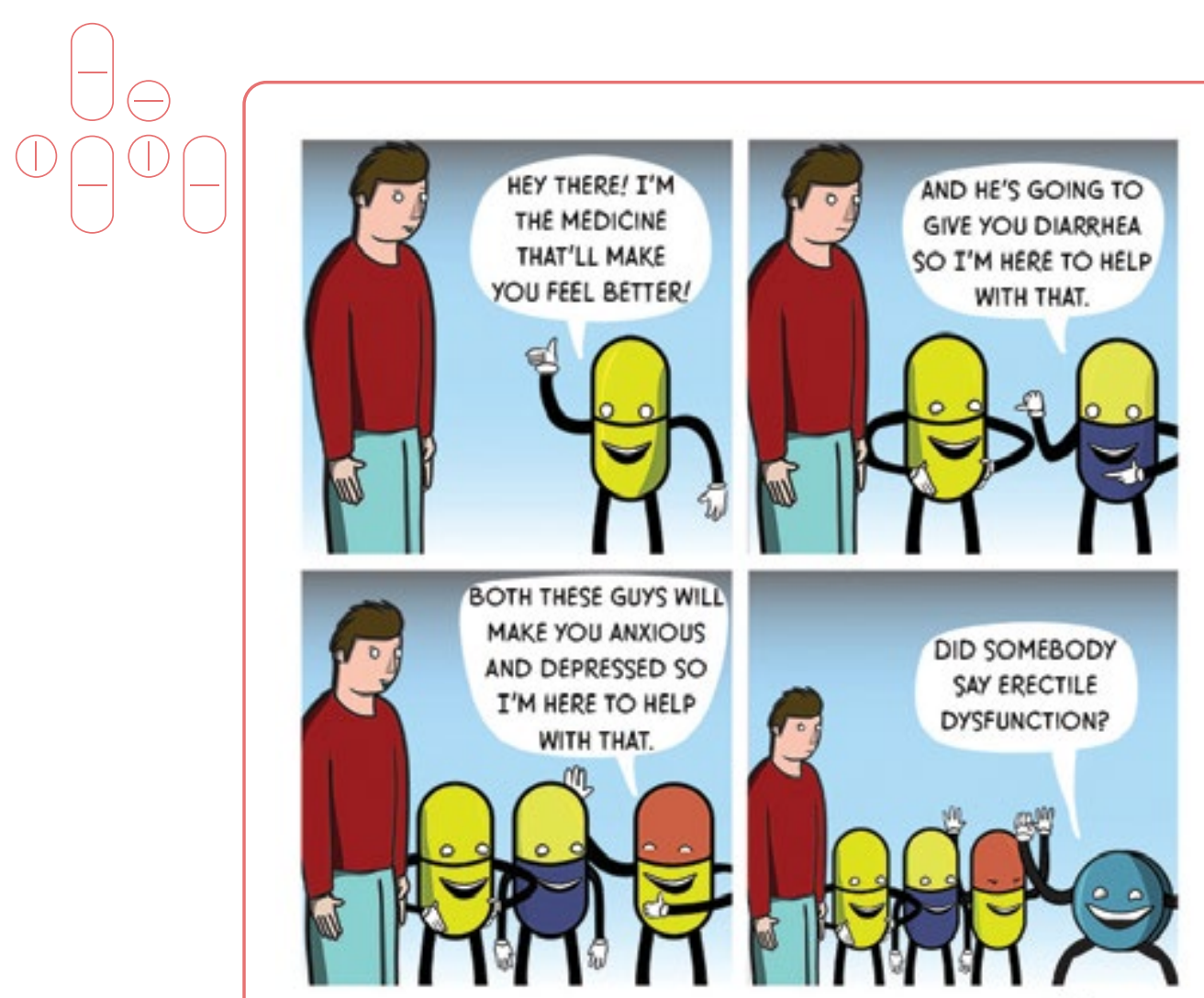


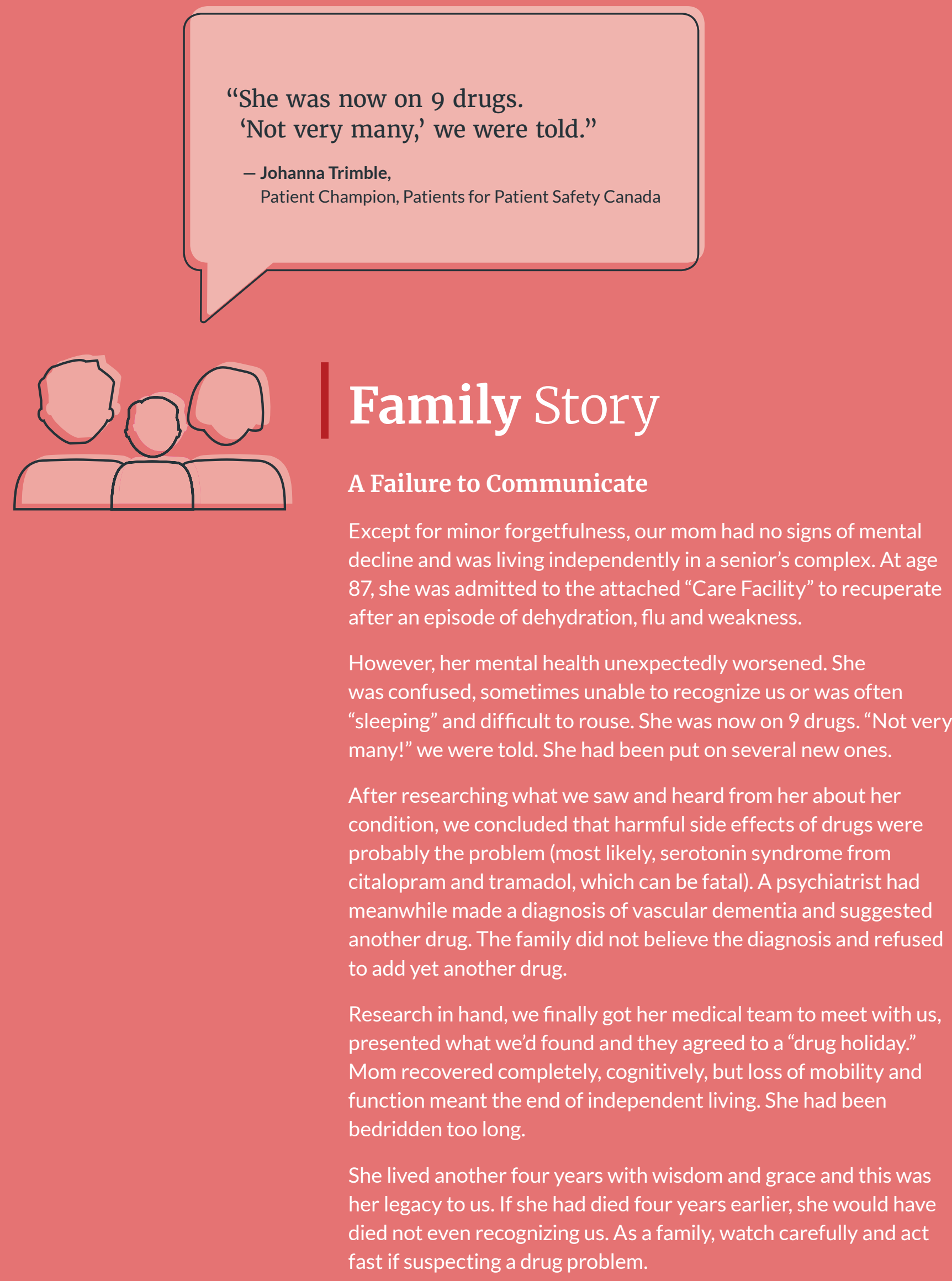




\section{Barriers to discontinuing or pausing medications}

Many of the factors in health care that foster excessive prescribing also hinder efforts to "deprescribe," or discontinue medications that are no longer necessary or potentially harmful. Experts generally agree that older adults who take one or more drugs on a long-term basis should have an annual review of their medications, or prescription checkup.

A prescription checkup involves (a) reviewing a patient's list of medications and the reasons they were prescribed and (b) reducing the dose, or stopping or pausing those that are ineffective or harmful. In practice, prescription checkups and efforts to deprescribe have been extraordinarily difficult to implement, often because the factors that promote excessive prescribing also serve as barriers to discontinuing medications. For example:

\section{Lack of awareness}

The most significant barrier to prescription checkups and addressing medication overload is many health care professionals are simply unaware of the prevalence of the problem. ${ }^{33,34}$ They anticipate benefits and miss side effects, even serious side effects such as delirium and dizziness, which can masquerade as symptoms of underlying medical conditions.

\section{Lack of time and information}

Primary care clinicians are often reluctant to review patients' medications because it takes time, for which they are often inadequately compensated, especially for patients with multiple chronic illnesses, multiple physicians, and numerous prescriptions. ${ }^{55,64}$ Clinicians who do take the time are hindered by inadequate information. When patients bring their medications in for review (sometimes called a "brown bag" session), the health care professional often does not know when the patient started taking the medication or for what condition, because this information is not printed on the medication label and is not readily available in the EMR. ${ }^{69}$

\section{Fear of causing harm or discomfort}

Many clinicians fear that discontinuing medications will cause harm. They know that some drugs, such as opioids and benzodiazepines, need to be tapered, but they may be unfamiliar with the process. In addition, primary care clinicians are often reluctant to discontinue a drug previously prescribed by a specialist. They also worry that discontinuing a medication can be misinterpreted by patients as "giving up." ${ }^{44,64,70}$ 


\section{Interventions to address}

\section{medication overload}

The medication overload afflicting millions of older adults is a complex problem, with many causes and agents and no easy fix.

As such, we need a holistic, multi-pronged set of policies, regulations, and payment models, as well as changes in both the training and practice habits of health care professionals. All payers, but particularly Medicare, Medicaid, private insurers, and the Veterans Administration, have a role to play in tackling this problem. A comprehensive set of solutions must include interventions to help prevent excessive prescribing as well as interventions to promote judicious discontinuation of medications that are inappropriate, potentially harmful, or no longer necessary.

Solutions must address the three main drivers described previously: the culture of prescribing, information and knowledge gaps, and our fragmented health care system. In this section, we discuss current efforts and potential interventions to reduce the number of people put at risk by taking too many prescription medications. 


\section{Impact and Feasibility of Interventions}

\section{Culture of Prescribing Impact Feasibility}

Launch public service campaigns for both health professionals and non-professionals to increase awareness of medication overload

Empower patients and families by promoting the use of patient decision aids and shared decision making

Reduce pharmaceutical industry influence by limiting industry marketing to health professionals and direct-to-consumer advertising

\section{8}

\section{Information and Knowledge Gaps}

Impact

Feasibility

Ensure that clinical guidelines take into account patient age and comorbidities, and whenever possible, include recommendations for stopping medications

Further develop \& disseminate deprescribing guidelines to help clinicians and pharmacists know how to deprescribe safely

Include training on appropriate prescribing and deprescribing for all students/trainees, as well as continuing medical education training for health professionals

Continue research on medication overload and deprescribing to fill research gaps

Implement team-based care models in hospitals and clinics, incorporating pharmacists into care teams when possible

Give primary care providers adequate time and information to do prescription checkups

Make electronic medical records more user-friendly and fully interoperable, so patients and providers can easily access a full list of patients' medications

Implement patient-centered prescription checkups periodically and during care transitions 


\section{Address the Culture of Prescribing}

Transforming the culture of prescribing - where clinicians and patients reach for a pill as the easiest solution - is no easy task. Ultimately, we need to bring this crisis into the open by raising public and professional awareness, countering the influence of the pharmaceutical industry, and changing the way patients and their clinicians make decisions regarding treatment choices.

Public health campaigns aimed at increasing awareness of the dangers of excessive prescribing are needed to foster conversations among health care professionals and with their patients and families. Both the Centers for Disease Control and Prevention, the nation's premier public health institute, and the Centers for Medicare and Medicaid Services (CMS), as the nation's biggest payer, have a role to play in campaigns directed at the public, patients, families, and at health care professionals. Health systems and private insurers could also create awareness campaigns for their patients. The overprescribing of medications and the harms arising from it is a public health problem on the scale of the harms of tobacco smoking and considerable resources and time will be needed to address it.

Several groups and institutions have conducted campaigns to increase patient awareness of the risks associated with taking multiple medications and encourage conversations about deprescribing. The EMPOWER campaign, conducted by researchers at the University of Montreal and the Canadian Deprescribing Network, mailed informational brochures to older patients taking a benzodiazepine to encourage them to ask their doctor about tapering. The campaign led to a 22 percent greater reduction in benzodiazepine use among older adults after six months, compared to a control group. ${ }^{74}$

Public health campaigns will be more successful in changing prescribing behaviors if they also engage pharmacists and clinicians. ${ }^{74,76}$ In the EMPOWER study, 33 percent of patients who did not taper their medications reported being discouraged in doing so by their doctor or pharmacist. ${ }^{74,77}$ Teaching young prescribers some basic principles for conservative prescribing could also reduce medication overload. ${ }^{96}$ 


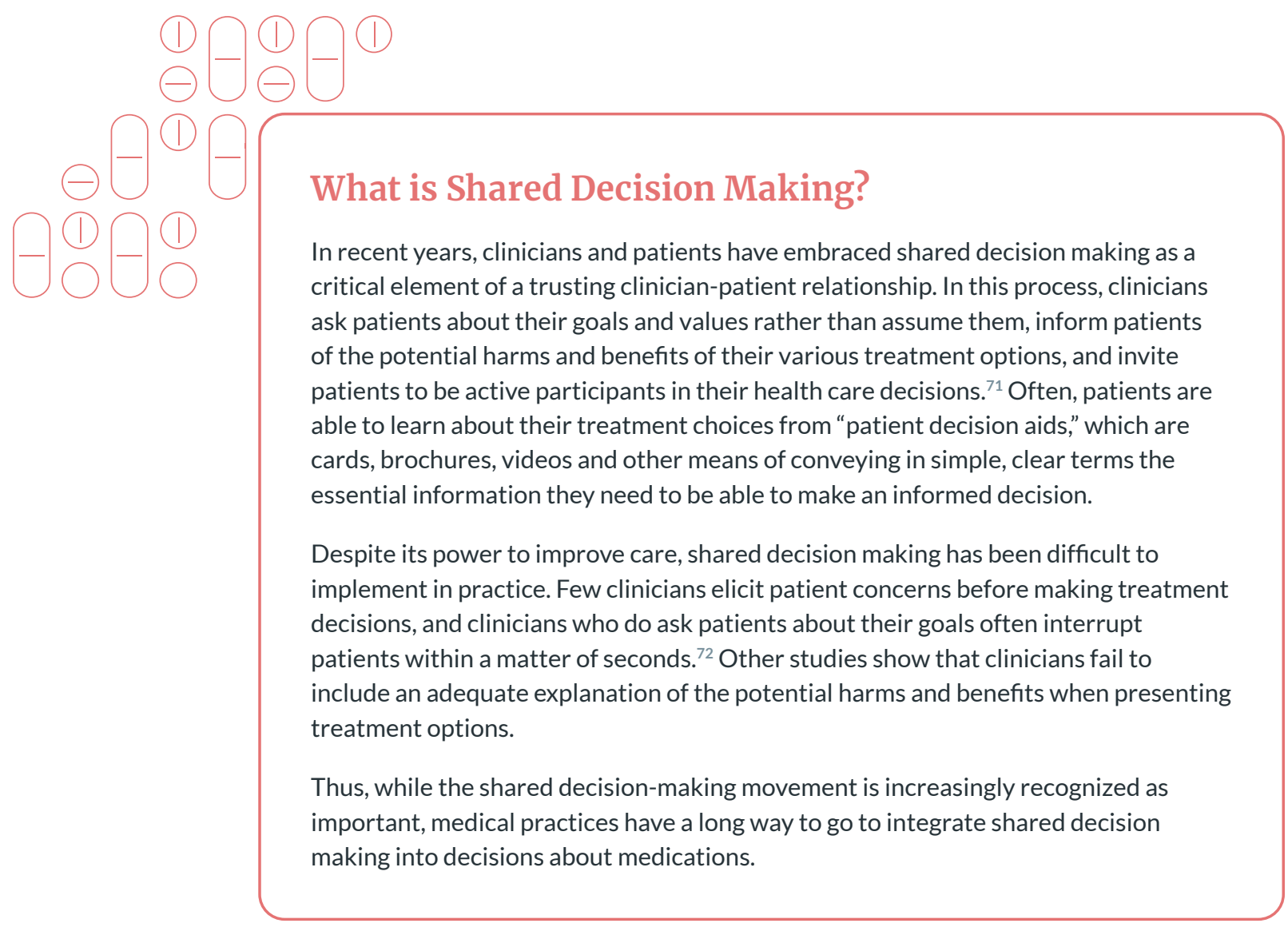

Empower patients and families
Patients and families are the ones most directly affected by prescribing decisions, yet too often health care professionals make treatment decisions unilaterally, without exploring the patient's goals, values, or preferences, or explaining the harms and benefits. ${ }^{72}$ Similarly, patients and families are often the first to recognize a side effect that affects a patient's ability to function, but their observations may be dismissed or minimized by health care professionals.

Patients must be empowered to share questions and concerns regarding medications with their clinicians. Questions such as "When will we know the drug works?" and "How will we know when to stop it?" should be routine. Clinicians, in turn, should welcome such discussions. This practice, known as shared decision making (see What is shared decision making?), must be integrated into health care practices through medical training and continuing education. In addition, decision aids for both patients and clinicians should be created, validated, and made widely available. ${ }^{78}$ 


\section{Reduce pharmaceutical industry influence}

The pharmaceutical and biotech industries strongly influence available evidence, research priorities, clinical guidelines, FDA regulation, and health care professionals' prescribing practices. They also influence patient and family perceptions of medications and drive demand for drugs that may not be appropriate. Many of the interventions that are needed to combat medication overload and promote prescription checkups would be more effective if they were coupled with efforts to counterbalance industry's influence. For example, in order to improve information at the point of care, medical societies creating clinical guidelines need to seek out sources of unbiased clinical evidence, preferably based on studies not funded by industry.

Short of banning direct-to-consumer advertising, there are ways to better inform consumers. For example, the FDA, which regulates drug advertising and testing, could require drug ads to explicitly state that medications have not been tested in those over the age of 65 . The agency could also require pharmaceutical advertisements to include data on the absolute, as opposed to relative, benefit of the drug. Finally, the FDA could mandate that Phase III drugs trials include testing in older adult populations.

\section{"Some of the medications being advertised} on television are weapons-grade drugs, with so many serious side effects they should only be rarely prescribed. No patient should be 'asking their doctor' about them."

- Alan Cassels

Journalist and author, Therapeutics Initiative, University of British Columbia 


\section{Fill Information and Knowledge Gaps}

\section{Improve information at the point of care}

Physicians and other clinicians need better, more evidence-based clinical practice guidelines, both for prescribing and for conducting prescription checkups and discontinuing medications. Currently, most guidelines are developed by medical specialty societies and professional associations without input from primary care physicians, who are often under pressure to follow them. Involving primary care physicians in the development and writing process would help to improve the utility of these guidelines.

Guidelines need to provide clinicians with a clear and credible synopsis of the best available evidence, presented in a way that allows them to discuss risks, benefits, and harms of potential treatments with patients. (The Choosing Wisely initiative is one such example of balanced, evidence-based guidelines.) Prescribing guidelines should incorporate information on appropriate doses for older patients and stronger cautions against increasing medications prescribed for these patients.

International institutions, such as the National Health Service of Scotland and the Bruyére Research Institute in Ottawa, Ontario, have begun developing guidelines for deprescribing. ${ }^{51,79}$ These guidelines should be widely distributed, along with yet-to-be-developed deprescribing guidelines for all drug classes that are commonly overprescribed or implicated in ADEs. Those publishing deprescribing guidelines must also recognize and address the pervasive influence of drug manufacturers on clinician beliefs and the scientific evidence.

\section{Changes to clinical practice guidelines?}

Several key changes to clinical practice guidelines could help to eliminate bias toward the "average patient" and allow prescribers to better weigh the harms and benefits of medications for older patients. These include:

- Warnings of side effects for prescribing for older adults or those with specific chronic conditions

- Warnings regarding adverse drug reactions common for those taking multiple medications

- Information about when doctors should taper or stop medications 


\section{Educate and train health care professionals to avoid medication overload}

With an aging population, all medical, nursing, and pharmacy students need greater competency in geriatric care. Especially important is understanding the risks of multiple medications for older adults with chronic conditions. This should be a priority for medical schools and health care accrediting bodies.

Additionally, medical, nursing, and pharmacy curricula need to incorporate explicit instruction in judicious prescribing, including using lower doses for older patients. Curricula must also help students learn to stop or pause medications safely by introducing them to evidence-based tools and methods of deprescribing (see Appendix B for a list of evidencebased prescribing \& deprescribing tools, p. 44). Post-graduate continuing education should also introduce and reinforce these skills.

Some universities are making these changes. For example, the University of British Columbia in Vancouver, Canada, includes prescription risks and deprescribing in the curriculum for first-year medical students and second-year pharmacy doctoral students. ${ }^{48}$

Many aspects of medication overload are poorly understood and require more research. A research agenda should include the financial impact of overmedication, the rates of harm associated with medication overload separate from the harm from multiple chronic conditions, the risks and benefits of deprescribing specific drugs in older adults, and evaluations of best practices (see Gaps in the research: Unanswered questions). To avoid conflicts of interest, this research needs public support, including funding through public/private partnerships that do not involve the pharmaceutical industry.

\section{Gaps in the research: Unanswered questions}

- What proportion of adverse events and mortality is caused by polypharmacy, given that polypharmacy correlates with age and comorbidity? What are the harms of polypharmacy when we adjust for patient risk?

- How should prescribers adjust doses for medications used by older adults and for the subpopulation of older adults with frailty and multimorbidity ${ }^{51}$

- What are the risks and benefits of deprescribing certain drugs? Does deprescribing reduce the rate of adverse events in older adults? What are the potential negative consequences of deprescribing? ${ }^{51}$

- For what drugs and in what populations does deprescribing work best?

- What are the most effective interventions for changing clinician behavior and facilitating prescription checkups?

- How can we successfully engage multiple stakeholders (doctors, nurses, pharmacists, patients, and families) in deprescribing initiatives simultaneously?

- What are the potential financial savings of deprescribing - for the patient and for the health system as a whole? 


\section{Reduce Fragmentation of Care}

\author{
Foster \\ communication \\ and \\ coordination \\ of care
}

Innovations in health care delivery, such as integrated health systems and team-based models of care, are becoming more common and offer tremendous potential for improving care coordination and reducing excess prescribing. For example:

- The Program of All-Inclusive Care for the Elderly (PACE) provides an interdisciplinary team of health professionals for frail elders, to help them remain in the community rather than in the nursing home. Evaluations show that PACE participants take fewer medications overall and fewer anticholinergic medications than patients not in the program. ${ }^{80}$

- The Institute for Healthcare Improvement, in partnership with the John A. Hartford Foundation, has a new initiative called "age-friendly health systems," built around improving care for older adults by using evidencebased models across the care continuum. The program's " $4 \mathrm{M}$ " bundle, which comprises "What Matters, Medication, Mentation, and Mobility," provides a guide for avoiding excess prescriptions and for deprescribing. The bundle is being tested by health systems around the country.

- The Kaiser Permanente (KP) health system integrates hospitals, physicians, and health insurance under the same systemwide EMR and global budget. With medical care integrated across care sites and medical records available to all clinicians, the KP system has emerged as a leader in addressing medication overload. ${ }^{81}$

For integrated care teams to be effective, the primary care clinician (for a medication evaluation team, this also could be a pharmacist) must be the hub of a wheel, receiving all the necessary information and empowered to conduct regular prescription checkups and reduce dosages or discontinue medications as needed. To do this well, primary care clinicians or pharmacists need:

- Access to information about all the medications a patient is taking. EMRs have the potential to solve this problem but they must be fully interoperable and include not only patient's medications, but when the patient began taking the medications and the conditions for which they have been prescribed.

- Time to review the patient's goals and symptoms, and time to begin the process of tapering or discontinuing drugs that both patient and clinician agree are superfluous or potentially harmful.

- The ability to readily access support for non-pharmacological therapies. Sleeplessness, for example, can be treated using sleep hygiene techniques, such as avoiding stimulants close to bedtime and establishing a regular bedtime routine. EMR systems must be able to facilitate "prescribing" of these non-pharmacological therapies. 


\section{Implement routine prescription checkups}

At several points along the patient journey, whether it's during or after a stay in the hospital, during a stay in rehabilitation facility or nursing home, or during a primary care visit, health care professionals need to periodically review all the medications a patient is taking and consider whether or not to stop one or more current prescriptions. A prescription checkup can be done not only in hospitals and clinics, but at retail pharmacies as well. For example, in some Canadian provinces, pharmacists are paid $\$ 80-\$ 100$ by their provincial governments to conduct 30-40-minute meaningful medication reviews in older adults, though a 2016 evaluation found little evidence that pharmacists were deprescribing through this program yet. ${ }^{82}$

Health care professionals have developed several approaches to prescription checkups, such as the CEASE Protocol, the Medication Appropriateness Index and the 10-step discontinuation guide (see Appendix B for a longer list of deprescribing tools, p.44).51,83 These approaches are not as black and white as explicit "bad drug" lists (e.g., Beers Criteria), allowing clinicians and patients to take clinical circumstances and patient preferences into consideration during the decision-making process. These models show promise in reducing adverse drug events and the number of medications prescribed for older adults with multiple chronic conditions. However, they require a greater time commitment and more knowledge from practitioners than do lists of drugs to avoid..$^{51}$

\section{U.S. public policies related to medication overload}

Accountable Care Organizations (ACOs)

As a result of the Affordable Care Act, nearly 500 ACOs are now serving 10 million Medicare and Medicaid patients. Clinicians, hospitals, and other health care professionals in an ACO are jointly responsible for their patients' health and are rewarded for meeting quality targets and avoiding unnecessary tests and procedures. ACOs provide many elements necessary for deprescribing, including electronic prescription transmissions and integrated pharmacy and medical records, and some ACOs that incorporate pharmacists have been successful at reducing inappropriate medications. ${ }^{84,85}$ However, most currently operating ACOs do not include pharmacists, and thus far, evidence does not indicate that ACOs as a whole are reducing excessive prescribing. ${ }^{85-87}$

\section{Medication therapy management}

Since 2006, the Center for Medicare and Medicaid Services (CMS) has required that all Part D plans include Medication Therapy Management (MTM) programs. MTMs are designed with the laudable goal of helping "improve medication use, reduce the risk of adverse events, and improve medication adherence." ${ }^{87}$ However, these reviews are not designed specifically to reduce unnecessary medications, which may limit their effectiveness for deprescribing. ${ }^{89}$

\section{Reducing Anti-Psychotic Use in Nursing Homes}

In 2012, CMS launched an effort to reduce unnecessary antipsychotic use among nursing home residents with dementia. This multi-pronged initiative, including a national partnership of stakeholders and requirements to include antipsychotic use in public reporting, reduced antipsychotic use by 34 percent nationwide over five years. However, some research indicates that some nursing homes have replaced antipsychotics with other mind-altering medications. Changes in health outcomes such as falls and hospitalizations from the initiative have not been studied. ${ }^{83}$ 
"I never realized how much medication management could make a difference in my life until someone fixed it."

- Michael Manganiello

Partner at HCM Strategists

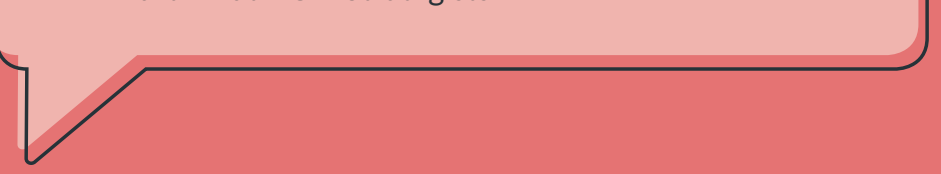

\section{Patient Story}

\section{Living with HIV, Managing Medications}

I never realized how much medication management could make a difference in my life until someone fixed it. I am a very active 60-year-old, and I am HIV positive so I have a cocktail of about 13 different medications I need to take each month. When my local community health center moved into a new building with an in-house pharmacy, my meds became impossible to manage. The prescribing times for my meds were out of sync, so I found myself having to go to the pharmacy every four days to refill one, spending nearly two hours a week in line.

One of my very effective antiviral medications was running out and it took me almost a week to be able to get it refilled. At that point I was practically in tears, begging the pharmacist to figure out the preauthorizations and refill the medication. The stress was starting to hurt my health and I was seriously considering leaving the health center.

Then the pharmacy created a new system, in which "highutilizers" like myself were given one pharmacist to manage the group. Soon after, my medication refill times were perfectly synced up, so I only have to go to the pharmacy once a month. This new system has changed my life. I'm not stressed out anymore and I got so many hours back. People shouldn't have to think about their meds all day. It's hard enough every morning and night swallowing a zillion pills. It makes all the difference that I don't have to worry about my meds. 


\section{A National Strategy for Addressing Medication Overload}

The U.S. has had limited success with a handful of policies related to medication overload (see U.S. public policies related to medication overload, p. 34), but we continue to lag behind other high-income countries that are doing much more to directly address medication overload. Canada and Australia, for example, have established "deprescribing networks" made up of researchers, clinicians, pharmacists, and patient advocates who have come together to share information, draft strategies, and disseminate proven interventions to help clinicians stop or pause potentially dangerous or unnecessary medications. ${ }^{90}$ The European Union's Health Program created a consortium of stakeholders from across the EU called Project SIMPATHY, to address the issue of excessive prescribing and medication non-adherence. ${ }^{79}$ The two-year project produced a systematic review of EU polypharmacy policies, analyses of potential actions, case studies, and a website/app with tools for conducting medication reviews. . $9,91^{2}$

While our country has woken up to the severity of the opioid crisis, medication overload has not yet been fully recognized as another drug epidemic in need of a national response. To catalyze action on this critical issue, the Lown Institute and a working group of expert clinicians, pharmacists, researchers, health policy advocates, and patients have undertaken a year-long effort to draft a National Action Plan for Addressing Medication Overload. This plan will focus on the most urgent actions needed to combat this epidemic and the paths to implementing them. The plan's recommendations will include both upstream interventions intended to prevent excessive prescribing, and downstream interventions aimed at promoting regular prescription checkups and helping patients and families as well as health care professionals pause or stop harmful or unnecessary medications.

The National Institutes of Health $(\mathrm{NIH})$ is reviewing applications for a multidisciplinary research network on deprescribing, which will be launched in 2019. This research is necessary and should be complemented by the establishment of a network of health care professionals, including clinicians, pharmacists and researchers, as well as patient and health reform advocates, who are committed to working together to drive rapid change. Such change is needed in current practices in acute care settings such as hospitals, doctors' offices, clinics, and long-term care settings such as care homes and skilled nursing facilities. Many health care professionals, patients, and advocates have expressed interest in establishing a North American medication overload network, but without funding and a means of collaborating, progress will be slow. 


\section{Conclusion}

If nothing is done to change current practices, medication overload will lead to the premature deaths of at least 150,000 older people in the U.S. over the next decade, and it will reduce the quality of life for millions more.

Strong, coordinated, and immediate action is needed to stem the tide of this epidemic, which harms people of all ages, but especially older generations. We must address the pervasive culture of "more is better" in medicine, conduct needed research to provide better information at the point of care, and light a path to minimizing the number of unnecessary and potential harmful drugs patients are taking.

This report should serve as a clarion call to policymakers, regulators and legislators, along with health care professionals and patient advocates, to come together to create, adopt, and implement a national strategy for addressing this crisis. Such a strategy can build on the recommendations in the National Action Plan for Addressing Medication Overload, which will be released early next year, to create a thoughtful and inclusive framework for systemic change that will produce measurable and meaningful results.

Focusing on reducing inappropriate or unnecessary medications could save at least $\$ 60$ billion over the next decade in unnecessary hospitalization for older adults alone. As a nation, we would also save billions more on the cost of unnecessary drugs and visits to the emergency room and outpatient clinics. More important even than the associated costs, successfully tackling the problem of medication overload holds the promise of lessening disability, cognitive decline, and time in the hospital for patients. That translates into better lives for millions of older people. 
38 Medication Overload: America's Other Drug Problem | Lown Institute 


\section{Appendices and References}

APPENDIX A:

Calculating the Harm from

Medication Overload

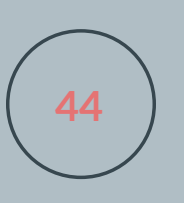

APPENDIX B

Evidence-Based Prescribing \& Deprescribing Tools

References 


\section{Appendix A: Calculating the harm from medication overload}

To calculate estimates of harm from adverse drug events, we relied primarily on two sources:

- Shehab N, Lovegrove MC, Geller A. US Emergency Department Visits for Outpatient Adverse Drug Events, 2013-2014. JAMA 2016; 316(20): 2115-25.

- Bourgeois FT, Shannon MW, Valim C, Mandl KD. Adverse drug events in the outpatient setting: an 11-year national analysis. Pharmacoepidemiology and drug safety 2010; 19(9): 901-10.

These studies provide estimated rates of harm from ADEs for older adults in the U.S. The study by Shehab et al. includes estimated rates of emergency department (ED) visits and hospitalizations from ADE for older adults from 2005-2014. The study by Bourgeois et al. includes estimated rates of outpatient visits for ADE for older adults from 1995-2005. We acknowledge that the latter study relies on older data and may not be exactly representative of current practice. However, this was the best source we found that included the most recent estimate of outpatient visits caused by ADEs.

We chose these studies because they both use measurements of ADE that seek to capture harm caused by medications that were prescribed to patients, rather than simply hospital or outpatient visits with any ADE code present on admission. In the study by Shehab et al., the rate of adverse events was determined through a search of clinical records of every ED visit to find any "clinician-diagnosed adverse drug events that are the reason for the ED visit." In the study by Bourgeois et al., the authors measured the rate of "all ADE-related visits" using the surveys. The survey data for each visit include up to three reasons for patient visit, up to three physician diagnoses for visit, and "whether the visit is related to adverse effects of medical treatment, including adverse drug events." Both studies omitted ADEs caused by intentional overdose or use of illicit substances.

Table 1 below shows the estimated rates of harm from ADEs using data from Shehab et al and Bourgeois et al. 95\% confidence intervals are provided. 


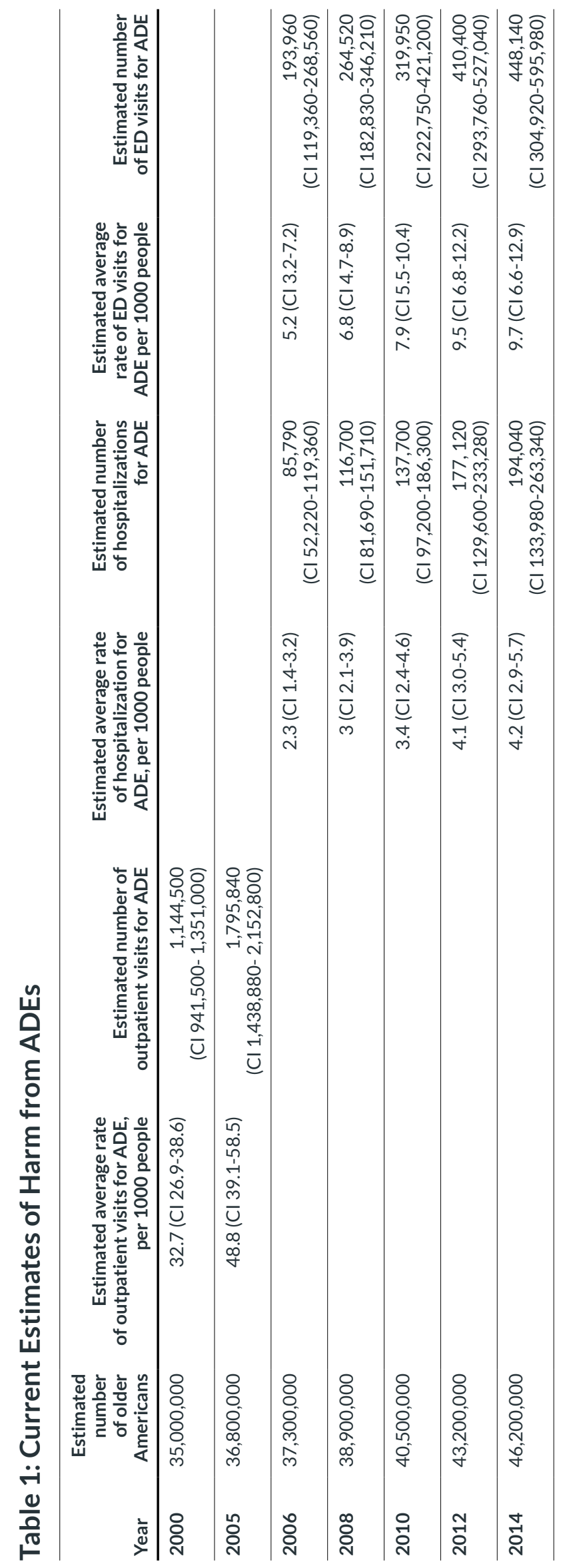

(41) Medication Overload: America's Other Drug Problem | Lown Institute 
In Table 2 (p. 43), we use the estimated rates of harm from the previously described sources to calculated estimated rates of harm in the future. Making these predictions required us to make a few assumptions. The rate of ED visits for ADE greatly increased between 2006 and 2012, but slowed suddenly between 2012 and 2014, the two most recent years we have data. We do not know whether this is an anomaly within a larger pattern of increasing harm from drug use, or whether it is the beginning of a slowdown in harm due to better safety procedures. To that end we provide two projections, one in which the pattern from 2006-2014 continues and ADEs increase at a greater rate (the "high estimate" in the chart), and one which shows a slower rate of increase commensurate with the last two years of available data (the "low" estimate in the chart).

For ED visits and hospitalizations, we ran two different regressions, one using all data points provided from 2006-2014, and one using just the final two data points provided. For outpatient visits, we ran one regression using the most recent data points provided in Bourgeois et al. For the $\mathrm{x}$-values, we used the last one or two digits of the year (2012 became 12, for example). The trendline equations are included in the chart as well as $95 \%$ confidence intervals where applicable.

For estimates of U.S. population and population over the age 65, we used Census projections and estimates, and reports from the Health and Human Services (HHS) Administration on aging. ${ }^{92-95}$ There were no estimates available for years between 2020 and 2030 except for 2025 .

For estimates of cost and mortality from ADE hospitalizations, we used an analysis of adverse drug events in U.S. hospitals from the Hospital Cost and Utilization Project (Weiss AJ, Freeman WJ, Heslin KC, Barrett ML. Adverse Drug Events in U.S. Hospitals, 2010 Versus 2014. HCUP Statistical Brief \#234. Rockville, MD: Agency for Healthcare Research and Quality; 2018.)

Weiss et al. found that the rate of mortality for adverse drug events present on admission was 3.2 percent in 2014 (for all ages). We applied this mortality rate to estimate the number of deaths from ADE-related hospitalizations (this is for all ages, so may underestimate mortality from ADE for older Americans). The average cost per hospitalization with ADE was $\$ 13,308$. We acknowledge that the definition of ADE in Weiss et al. is different from that in the sources previously used; however, this was the only source we found that provided cost and mortality estimates from adverse drug event hospitalizations.

We believe that the "high estimate" scenario is more likely given that multiple medication use among older Americans has continued to increase over the past decade, which is why we have cited these estimates in the report. However, it is worth noting that our "low" estimate still predicts a serious 3 million hospitalizations from 2020-2030 at a cost of 100,000 lives and more than $\$ 40$ billion.

We believe that more research needs to be done on projecting the rate of harm from ADEs and hope this preliminary analysis can help inform that future work. 


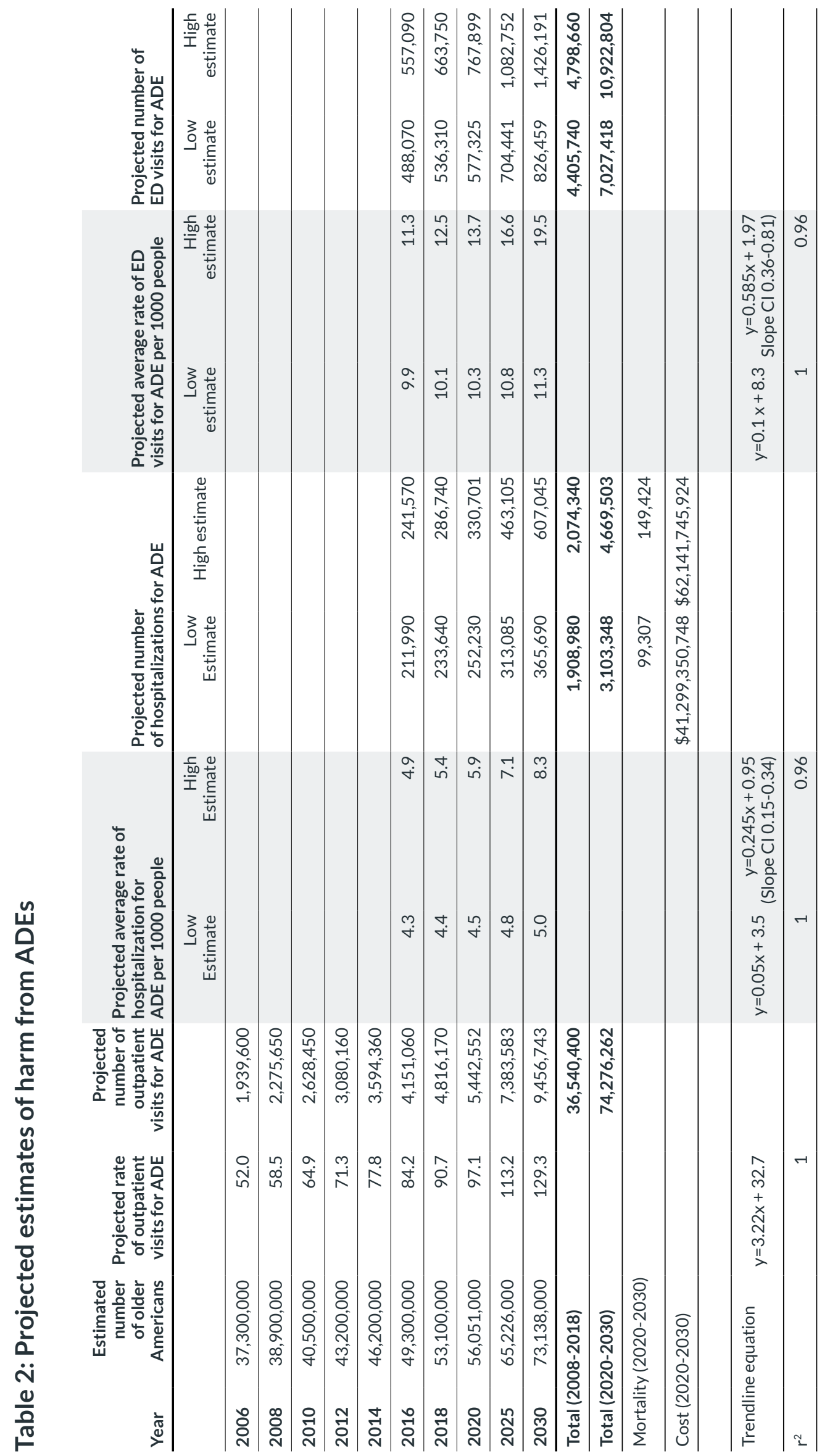

(43) Medication Overload: America's Other Drug Problem / Lown Institute 


\section{Appendix B: Evidence-based prescribing \& deprescribing tools}

\section{Tools for careful prescribing}

- 10-step framework for minimizing inappropriate medications A conceptual framework for quality use of medicine, to reduce inappropriate prescribing. Developed in 2012 by clinicians at the Princess Alexandra Hospital in Brisbane, Australia.

- The Absolute CVD Risk/Benefit Calculator A tool to assess patient risk of cardiovascular disease, developed by James McCormack and a group of family physicians called the PEER team.

- Beers Criteria Criteria developed by a 13-member expert panel from the American Geriatric Society. Contains a list of drugs to avoid, drug-disease combinations to avoid, drugs to use with caution in renal impairment, and drugs with strong anticholinergic properties; does not contain information on alternative medications.

- Best Science Medicine Podcast A podcast created by Dr. James McCormack and Dr. Michael Allan, to promote healthy skepticism and critical thinking, with a casebased approach.

- Choosing Wisely guidelines

Recommendations developed by the American Board of Internal Medicine Foundation with medical specialty societies to identify and reduce commonly overused tests and procedures.

- The C-TOP Tool

A tool for comparing treatment options for pain, developed by the PEER team along with the Physician Learning Program and the Alberta College of Family Physicians.

\section{- Medication Appropriateness Index} An implicit prescribing quality measure developed in 1992 by Dr. Joseph Hanlon and colleagues at the Center for the Study of Aging and Human Development, Duke University Medical Center.

- The NNT

Quick evaluations of the costs and benefits of common medications and therapies in an easy-to-understand format, by a group of independent physicians.

- PADIS Guidelines

Evidence-based, patient-centered guidelines developed to prevent and manage pain, agitation, delirium, immobility, and sleep disruption in the ICU, developed by the Society of Critical Care Medicine.

- Principles for more conservative prescribing Evidence-based, patient-centered prescribing principles that can avoid medication overload.

- Primary Health Tazmania Medicine Use Guides

A clinical tool with short guides to the use of medicines in older people for antidepressants, antipsychotics, and benzodiazepines.

- Simplified lipid guidelines

A guideline for for lipid management and prevention of cardiovascular disease, written by physicians with no industry support.

- The SPARC Tool

A great tool for going over the risks and benefits of preventive treatments for stroke, developed by Dr. Peter Loewen.

\section{- START and STOPP criteria for potentially} inappropriate prescribing

If/then indicators for starting medication or stopping medication, developed by Dr. Denis O'Mahony and colleagues from the University of Cork, Ireland. 


\section{Tools for deprescribing}

- Canadian Deprescribing guidelines

Evidence-based guidelines for deprescribing for five classes of medications: Proton pump inhibitors, Antihyperglycemic, Antipsychotic, Benzodiazepine Receptor Agonist, and Cholinesterase Inhibitors (ChEIs) and Memantine. Each guideline is accompanied by a decision-support algorithm, patient pamphlet, infographic and for some, a whiteboard video on how to use the algorithm. These guidelines were developed by researchers at the Bruyère Research Institute Deprescribing Guidelines Research Team and the Université de Montréal.

\section{- CEASE protocol}

Developed by members of the Australian Deprescribing Network.

- Good Palliative-Geriatric Practice Algorithm Developed by Dr. Doron Garfinkel and Dr. Dee Mangin, for deprescribing in elderly nursing home patients and community-dwelling older adults.

\section{- MedSafer}

An electronic deprescribing tool that could help physicians and pharmacists through the process of safe and successful medication deprescription in older adults. Cross-references lists of patient conditions with home medication lists to provide electronically generated deprescribing reports based on Beers' criteria, STOPP rules, and Choosing Wisely recommendations. Available to link with EMRs and for use in nursing homes or long-term care facilities. Developed by independent Canadian researchers.

Contact for different formats: emily.mcdonald@medsafer.org.

\section{- MedStopper}

An online tool to help clinicians and patients make decisions about reducing or stopping medications, based on their potential to improve symptoms, reduce the risk of future illness, and likelihood of causing harm. This tool is funded by a Knowledge Translation grant from the Canadian Institute of Health Information and is administered through the University of British Columbia.

\section{- PPI patient decision aid}

A tool to help patients decide whether they should keep taking their proton pump inhibitor (acid reflux medication), based on the Bruyère Research Institute Deprescribing Guideline.

\section{- Practical guide to stopping medicines in} older people

An article published in Best Practice Journal that contains specific guidance on safe tapering methods for specific drug classes

\section{- Primary Health Tazmania Deprescribing guides}

Short deprescribing tools for clinicians for $10+$ drug classes, written by pharmacist Dr. Peter Tenni and geriatrician Dr. David Dunbabin, in consultation with a deprescribing clinical reference group for Primary Health Tazmania.

\section{- SIMPATHY Project}

A website containing resources from the European Union's SIMPATHY Project, a twoyear project studying polypharmacy, led by the Scottish government. 


\section{References}

1 Shehab N, Lovegrove MC, Geller A. US Emergency Department Visits for Outpatient Adverse Drug Events, 2013-2014. JAMA 2016; 316(20): 2115-25.

2 Bourgeois FT, Shannon MW, Valim C, Mandl KD. Adverse drug events in the outpatient setting: an 11-year national analysis. Pharmacoepidemiology and drug safety 2010; 19(9): 901-10.

3 HCUP Fast Stats. Healthcare Cost and Utilization Project (HCUP) 2018. www.hcup-us.ahrq.gov/ faststats/opioid/opioiduse.dio3=on\&location1=US\&ch aracteristic1 $=01 \&$ setting $1=E D \&$ location $2=U S \&$ charact eristic $2=01 \&$ setting $2=I P \&$ expansionInfoState $=$ hide $\& d$ ataTablesState=hide\&definitionsState $=$ hide\&exportS tate=hide.

4 Statistics NCfH. Health, United States, 2016: With Chartbook on Long-term Trends in Health. In: Statistics NCfH, editor.; 2017.

5 Qato DM, Wilder J, Schumm LP, Gillet V, Alexander GC. Changes in Prescription and Over-the-Counter Medication and Dietary Supplement Use Among Older Adults in the United States, 2005 vs 2011. JAMA Intern Med 2016; 176(4): 473-82.

6 Masnoon N, Shakib S, Kalisch-Ellett L, Caughey GE. What is polypharmacy? A systematic review of definitions. BMC geriatrics 2017; 17(1): 230.

7 Hanlon JT, Pieper CF, Hajjar ER, et al. Incidence and predictors of all and preventable adverse drug reactions in frail elderly persons after hospital stay. J Gerontol A Biol Sci Med Sci 2006; 61(5): 511-5.

8 Viktil KK, Blix HS, Moger TA, Reikvam A. Polypharmacy as commonly defined is an indicator of limited value in the assessment of drug-related problems. Br J Clin Pharmacol 2007; 63(2): 187-95.

9 Gandhi, Weingart, Borus, et al. Adverse drug events in ambulatory care. New England Journal of Medicine 2003; April 2017(348).

10 Hein C, Forgues A, Piau A, Sommet A, Vellas B, Nourhashemi F. Impact of Polypharmacy on Occurrence of Delirium in Elderly Emergency Patients. Journal of the American Medical Directors Association 2014; 15(11).
11 Maher RL, Hanlon J, Hajjar ER. Clinical consequences of polypharmacy in elderly. Expert opinion on drug safety 2014; 13(1): 57-65.

12 Zia A, Kamaruzzaman S, Tan M. Polypharmacy and falls in older people: Balancing evidence-based medicine against falls risk. Postgraduate Medicine 2014; 127(3).

13 Dhalwani N, Fahami R, H S, S S, MJ D, K K. Association between polypharmacy and falls in older adults: a longitudinal study from England. BMJ open 2017; 7(10).

14 Leelakanok N, Holcombe AL, Lund BC, Gu X, Schweizer ML. Association between polypharmacy and death: A systematic review and meta-analysis. Journal of the American Pharmacists Association: JAPhA 2017; 57(6): 729-38.e10.

15 Shehab N, Lovegrove MC, Geller A. US Emergency Department Visits for Outpatient Adverse Drug Events, 2013-2014. JAMA 2016; 316(20): 2115-25.

16 Lee T, Cavalcanti R, McDonald E, Pilote L, Brophy J. Diastolic Hypotension May Attenuate Benefits from Intensive Systolic Targets: Secondary Analysis of a Randomized Controlled Trial. The American journal of medicine 2018; 131(10): 1228-33.

17 Young SL, Taylor M, Lawrie SM. "First do no harm." A systematic review of the prevalence and management of antipsychotic adverse effects. Journal of psychopharmacology (Oxford, England) 2015; 29(4): 353-62.

18 Pottie K, Thompson W, Davies S, et al. Evidencebased clinical practice guideline for deprescribing benzodiazepine receptor agonists. . 2016.

19 Jong d, Elst Vd, Hartholt. Drug-related falls in older patients: implicated drugs, consequences, and possible prevention strategies. Ther Adv Drug Saf 2013; 4(4): 147-54.

20 Wright JM, Bassett K. How well do you know your anticholinergic (antimuscarinic) drugs? Therapeutics Letter. British Columbia, CA: Department of Pharmacology and Therapeutics, University of British Columbia; 2018. 
21 Gray S, Hanlon J. Anticholinergic medication use and dementia: latest evidence and clinical implications. Therapeutic Advances in Drug Safety 2016; 7(5): 217-24.

22 Claxton A, Cramer J, Pierce C. A systematic review of the associations between dose regimens and medication compliance. Clinical Therapeutics 2001; 23(8): 1296-310.

23 Farrell B, French Merkley V, Ingar N. Reducing pill burden and helping with medication awareness to improve adherence. Can Pharm J (Ott) 2013; 146(5): 262-9.

24 Mehegan L, Skufca L. 2015 Survey on Prescription Drug Costs. Washington, DC: AARP Research, 2016.

25 Soumerai S, Pierre-Jacques M, Zhang F. CostRelated Medication Nonadherence Among Elderly and Disabled Medicare Beneficiaries. JAMA Intern Med 2006; 166(17): 1829-35.

26 Kantor ED, Rehm CD, Haas JS, Chan AT, Giovannucci EL. Trends in Prescription Drug Use Among Adults in the United States From 1999-2012. JAMA 2015; 314(17): 1818-31.

27 Mitchell AA, Kaufman DW, Rosenberg L. Patterns of Medication Use in the United States. Boston, MA: The Sloane Epidemiology Center at Boston University, 2007.

28 Jokanovic N, Tan EC, Dooley MJ, Kirkpatrick CM, Bell JS. Prevalence and factors associated with polypharmacy in long-term care facilities: a systematic review. Journal of the American Medical Directors Association 2015; 16(6): 535 e1-12.

29 Nightingale, Hajjar E, Swartz K, Andrel-Sendecki J, Chapman A. Evaluation of a pharmacist-led medication assessment used to identify prevalence of and associations with polypharmacy and potentially inappropriate medication use among ambulatory senior adults with cancer. Journal of Clinical Oncology 2015.

30 Balducci L, Goetz-Parten D, Steinman M. Polypharmacy and the management of the older cancer patient. Annals of Oncology 2013; 24(7): vii36vii40.

31 Segal M, Rollins E, Hodges K, Roozeboom M. Medicare-Medicaid Eligible Beneficiaries and Potentially Avoidable Hospitalizations. In: Services CfMaM, editor.; 2014 .
32 Mather M, Jacobsen LA, Pollard K, M. Aging in the United States. Population Bulletin 2015; 70(2).

33 Scott IA, Anderson K, Freeman CR, Stowasser DA. First do no harm: a real need to deprescribe in older patients. The Medical journal of Australia 2014; 201(7): 390-2.

34 Anderson K, Stowasser D, Freeman C, Scott I. Prescriber barriers and enablers to minimising potentially inappropriate medications in adults: a systematic review and thematic synthesis. BMJ open 2014; 4(12): e006544.

35 Garfinkel D, Ilhan B, Bahat G. Routine deprescribing of chronic medications to combat polypharmacy. Ther Adv Drug Saf 2015; 6(6): 212-33.

36 Anderson C, McNab D. Quality improvement project using a care bundle approach on the management of the immediate discharge document (IDD) within a single general practice. BMJ quality improvement reports $2015 ; 4(1)$.

37 Schwartz L, Woloshin S. Medical Marketing in the United States, 1997-2016. JAMA 2019; 321(1): 80-96.

38 Klara K, Kim J, Ross J. Direct-to-Consumer Broadcast Advertisements for Pharmaceuticals: OffLabel Promotion and Adherence to FDA Guidelines. Journal of General Internal Medicine 2018; 33(5): 651-8.

39 Aiken KJ, Swasy J, Braman A. Patient and Physician Attitudes and Behaviors Associated With DTC Promotion of Prescription Drugs - Summary of FDA Survey Research Results Food and Drug Administration, 2004.

40 Birrer R, Tokuda Y. Medicalization: A historical perspective. Journal of General Family Medicine 2017; 18(2): 48-51.

41 Khullar D. A Profusion of Diagnoses. That's Good and Bad. The New York Times. 2018.

42 Moynihan R. The marketing of a disease: female sexual dysfunction. The BMJ 2005; 330(192).

43 Project PP. Persuading the Prescribers: Pharmaceutical Industry Marketing and its Influence on Physicians and Patients: Pew Charitable Trusts, 2013.

44 Zeitan J, Ross J, Atal I, et al. Postmarketing studies for novel drugs approved by both the FDA and EMA between 2005 and 2010: a cross-sectional study. BMJ open 2017; 7(12). 
45 Fickweiler F, Fickweiler W, Urbach E. Interactions between physicians and the pharmaceutical industry generally and sales representatives specifically and their association with physicians' attitudes and prescribing habits: a systematic review. BMJ open $2017 ; 7(8)$.

46 Hoffmann T, Del Mar CB. Clinicians' Expectations of the Benefits and Harms of Treatments, Screening, and Tests. JAMA Intern Med 2017; 177(3): 407-19.

47 Ni Chroinin D, Ni Chroinin C, Beveridge A. Factors influencing deprescribing habits among geriatricians. Age Ageing 2015; 44(4): 704-8.

48 Trimble J. Personal interview with Johanna Trimble. In: Brownlee S, Saini V, editors.; 2018.

49 Cruikshank M. Learning to be old : gender, culture, and aging. Lanham, MD: Rowman \& Littlefield Publishers 2013.

50 Ioannidis JPA, Stuart ME, Brownlee S, Strite SA. How to survive the medical misinformation mess. Eur J Clin Invest 2017; 47(11): 795-802.

51 Mangin D, Bahat G, Golomb BA, et al. International Group for Reducing Inappropriate Medication Use \& Polypharmacy (IGRIMUP): Position Statement and 10 Recommendations for Action. Drugs Aging 2018.

52 Gordon J. The under-representation of elderly patients in a problem-based medical school curriculum. Medical Teacher 2007; 29(8): 844.

53 Smith K, Ashburn S, Aminawung J, Mann M, Ross J. Physician clinical management strategies and reasoning: a cross-sectional survey using clinical vignettes of eight common medical admissions. $B M C$ health services research 2014; 14(176).

54 Boyd CM, Darer J, Boult C, Fried LP, Boult L, Wu AW. Clinical practice guidelines and quality of care for older patients with multiple comorbid diseases: implications for pay for performance. JAMA 2005; 294(6): 716-24.

55 Ailabouni NJ, Nishtala PS, Mangin D, Tordoff JM. Challenges and Enablers of Deprescribing: A General Practitioner Perspective. PLoS One 2016; 11(4): e0151066.

56 Field TS, Gurwitz JH, Avorn J, et al. Risk factors for adverse drug events among nursing home residents. Arch Intern Med 2001; 161(13): 1629-34.

57 Schott G, Pachl H, Ulrich L, Gundert-Remy U, Ludwig W, Lieb K. The Financing of Drug Trials by Pharmaceutical Companies and Its Consequences. Dtsch Arztebl Int 2010; 107(16): 279-85.
58 Ahn R, Woodbridge A, Abraham A, et al. Financial ties of principal investigators and randomized controlled trial outcomes: cross sectional study. The BMJ 2017; 365.

59 Lexchin J, Bero L, Djulbegovic B, Clark O. Pharmaceutical industry sponsorship and research outcome and quality: systematic review. The BMJ 2003; 326: 1167.

60 Norris S, Holmer H, Ogden L, Burda B. Conflict of interest in clinical practice guideline development: a systematic review. PLoS One 2011; 6(10).

61 Greenway T, Ross J. US drug marketing: how does promotion correspond with health value? The BMJ 2017; 357: 1855.

62 Warshaw G. Providing Quality Primary Care to Older Adults. Journal of the American Board of Familly Medicine 2009; 22(3): 239-41.

63 Cooper JA, Cadogan CA, Patterson SM, et al. Interventions to improve the appropriate use of polypharmacy in older people: a Cochrane systematic review. BMJ open 2015; 5(12): e009235.

64 Fried TR, Tinetti ME, Iannone L. Primary care clinicians' experiences with treatment decision making for older persons with multiple conditions. Arch Intern Med 2011; 171(1): 75-80.

65 Steinman M. Interview with Michael Steinman. In: Brownlee S, editor.; 2018.

66 Flamm H. "They Want Docile": How Nursing Homes in the United States Overmedicate People with Dementia: Human Rights Watch, 2018.

67 Koppel R. EMR Entry Error: Not So Benign 2009. https://psnet.ahrq.gov/webmm/case/199/emr-entryerror-not-so-benign.

68 Levinson J, Price B, Saini V. Death By A Thousand Clicks: Leading Boston Doctors Decry Electronic Medical Records. CommonHealth: WBUR; 2017.

69 Tarn D, Paterniti D, Kravitz R, Fein S, Wenger N. How Do Physicians Conduct Medication Reviews? Journal of General Internal Medicine 2009; 24(12): 1296-302.

70 Reeve E, To J, Hendrix I, Shakib S, Roberts MS, Wiese MD. Patient barriers to and enablers of deprescribing: a systematic review. Drugs Aging 2013; 30(10): 793-807.

71 Barry M, Edgman-Levitan S. Shared Decision Making - The Pinnacle of Patient-Centered Care. New England Journal of Medicine 2012; (366): 780-1. 
72 Ospina N, Phillips K, Rodriguez-Gutierrez R, et al. Eliciting the Patient's Agenda- Secondary Analysis of Recorded Clinical Encounters. Journal of General Internal Medicine 2018.

73 Redberg R. Failing Grade for Shared Decision Making for Lung Cancer Screening. JAMA Intern Med 2018.

74 Tannenbaum C, Martin P, Tamblyn R, Benedetti A, Ahmed S. Reduction of inappropriate benzodiazepine prescriptions among older adults through direct patient education: the EMPOWER cluster randomized trial. JAMA Intern Med 2014; 174(6): 890-8.

75 Steinman MA, Landefeld C. Overcoming inertia to improve medication use and deprescribing. JAMA 2018; 320(18): 1867-9.

76 Reeve E, Wolff JL, Skehan M, Bayliss EA, Hilmer $\mathrm{SN}$, Boyd CM. Assessment of attitudes toward deprescribing in older medicare beneficiaries in the united states. JAMA Internal Medicine 2018.

77 Martin P, Tamblyn R, Benedetti A, Ahmed S, Tannenbaum C. Effect of a pharmacist-led educational intervention on inappropriate medication prescriptions in older adults: The d-prescribe randomized clinical trial. JAMA 2018; 320(18): 1889-98.

78 Alston C, Berger Z, Brownlee S, Elwyn G. Shared Decision-Making Strategies for Best Care: Patient Decision Aids: National Academy of Medicine, 2014.

79 McIntosh J, Alonso A, MacLure K, et al. A case study of polypharmacy management in nine European countries: Implications for change management and implementation. PLoS One 2018; 13(4).

80 Covington L, McCarrell J, Hoerster N. Prevalence of Anticholinergic Medication Use in the Program of All-Inclusive Care for the Elderly. The Consultant pharmacist : the journal of the American Society of Consultant Pharmacists 2016; 31(3): 168-74.

81 The How and Why of Deprescribing. 2018; Online: The Institute for Healthcare Improvement; 2018.

82 Kolhatkar A, Cheng L, Chan F, Harrison M, Law M. The impact of medication reviews by community pharmacists. Journal of the American Pharmacists Association 2016.

83 Brandt NJ, Simoni-Wastila L. Practice and Policy/ Research Implications of Deprescribing on Medication Use and Safety in Older Adults. Public Policy \& Aging Report 2018; 28(4): 116-21.

84 Dubois R, Feldman M, Lustig A, et al. Are ACOs ready to be accountable for medication use? Journal of Managed Care Pharmacy 2014; 20(1): 17-21.
85 Amara S, Adamson R, Lew I, Slonim A. Accountable Care Organizations: Impact on Pharmacy. Hospital Pharmacy 2014; 49(3): 253-9.

86 Colla C, Lewis V, Beaulieu-Jones B, Morden N. Role of Pharmacy Services in Accountable Care Organizations. Journal of Managed Care Special Pharmacy 2015; 21(4): 338-44.

87 McWilliams J, Najafzadeh M, Shrank W. Association of Changes in Medication Use and Adherence With Accountable Care Organization Exposure in Patients With Cardiovascular Disease or Diabetes. JAMA Cardiology 2017; 2(9): 1019-23.

88 Chavez-Valdez A. CY 2019 Medication Therapy Management Program Guidance and Submission Instructions. In: Services CfMaM, editor. Baltimore, MD: Department of Health and Human Services; 2018.

89 Implementation of a Medication Therapy Management Intervention in Ambulatory Care Settings: The AHRQ Health Care Innovations Exchange, 2016.

90 Tannenbaum C, Farrell B, Shaw J, et al. An Ecological Approach to Reducing Potentially Inappropriate Medication Use: Canadian Deprescribing Network. Canadian journal on aging = La revue canadienne du vieillissement 2017; 36(1): 97-107.

91 Stewart D, Mair A, Wilson M, et al. Guidance to manage inappropriate polypharmacy in older people: systematic review and future developments. Expert opinion on drug safety 2017; 16(2): 203-13.

92 Annual Estimates of the Resident Population for Selected Age Groups by Sex for the United States, States, Counties and Puerto Rico Commonwealth and Municipios: April 1, 2010 to July 1, 2017: US Census Bureau, Population Division, 2018.

932017 National Population Projection Tables. In: Bureau TUC, editor.

94 A Profile of Older Americans, 2009. In: US Department of Health and Human Services AoA, editor.; 2010.

95 A Profile on Older Americans, 2007. In: US Department of Health and Human Services AoA, editor.; 2008.

96 Schiff GD, Galanter WL, Duhig J, Lodolce AE, Koronkowski MJ, Lambert BL. Principles of Conservative Prescribing. Arch Intern Med. 2011;171(16):1433-1440. 


\section{Acknowledgments}

The creation of this report, including research, analysis, and writing, was supported by the Gordon and Betty Moore Foundation. We are grateful to our GBMF program officers, Beth Metcalf and Julie Lawrence, without whose guidance and support this initiative would not have been possible. We are grateful to them and to all our funders.

This report was authored by Judith Garber, MPP, Lown Institute Health Care Policy and Communications Fellow; and Shannon Brownlee, MSc, Senior Vice President. It would not have been possible without the hard work of the entire Lown project team - Carissa Fu, Julia Healey, Vikas Saini, and Aaron Toleos. Thanks to Karen Kahn for editing the report; Noam Bar-Zemer for leading the branding and focus group research with Kimberly Noel and KarDav Solutions; Ann Harakawa and her team at Two Twelve for conceptual design work. Thanks to Lown interns Niki Cozzolino and Doly Han for additional research.

We gleaned valuable information from the experiences of clinicians, academics, patient advocates, and others who encounter medication overload every day. Thanks to the following individuals for agreeing to be interviewed and sharing their insights:

Caleb Alexander, MD Associate Professor of Epidemiology and Medicine, co-director of the Center for Drug Safety and Effectiveness, Johns Hopkins University

Jerry Avorn, MD

Professor of Medicine, Harvard Medical School Chief of the Division of Pharmacoepidemiology and Pharmacoeconomics, Brigham and Women's Hospital

Ken Boockvar, MD

Professor of Geriatrics and Palliative Medicine, Mount Sinai Hospital

Cynthia Boyd, MD, MPH Professor of Medicine, Johns Hopkins University

Theresa Brown, BSN, RN Clinical Nurse/Author

Alan Cassels

Communications Director, University of British Columbia; journalist

Jamie Chan, PharmD Senior Director, Medicare Star Program, Blue Shield of California
Chris Chang, PharmD

Pharmacy Project Manager,

Kaiser Permanente, Northern

California

Lynn Deguzman, PharmD, BCGP

Regional Clinical Operations

Manager, Kaiser Permanente, Northern California

Barbara Farrell, PharmD

Clinician Investigator,

Bruyère Research Institute,

University of Ottawa

Joe Graeden, MS

Executive Producer and cohost, The People's Pharmacy

Emily Hajjar, PharmD, MS Thomas Jefferson University, Associate Professor of Pharmacy Practice

Laura Hanson, MD, MPH Professor of Geriatric

Medicine, Associate Director of Geriatric Fellowship Program, University of North Carolina

Corinne Hohl, MD

Associate Professor, Emergency Medicine, University of British Columbia

Holly Holmes, MD, MS, Division Director, Associate Professor, Geriatric and Palliative Medicine, McGovern Medical School
Aaron Kesselheim, MD

Associate Professor of

Medicine, Harvard Medical

School

Jean Kutner, MD, MPH/

MSPH

Professor of Medicine,

University of Colorado

Andy Lazris, MD

Primary care physician,

Personal Physician Care

Todd Lee, MD, MPH

Associate Professor of

Medicine, McGill University

Sei Lee, MD, MAS

Associate Professor, University of California, San Francisco

Sharon Levine, MD

Board of Directors, School of

Medicine, Kaiser Permanente

Amy Linsky, MD, MSc

Assistant Professor of

Medicine, Boston University;

Physician, VA Boston

Healthcare System

Claes Magnus Lundgren

Author of Fas Ut ("Phase Out")

Alpana Mair, MSc

Head of Effective Prescribing and Therapeutics, The Scottish Government
Dee Mangin, MBCHB

Professor of Family Medicine, McMaster University

James McCormack, PharmD

Professor of Pharmaceutical

Sciences, University of British Columbia

Emily McDonald, MD, MSc

Assistant Professor of Medicine, McGill University Health Centre

Thomas Moore

Senior Scientist, Drug Safety and Policy, Institute for Safe

Medication Practices

Dima Qato, PhD, PharmD, MPH

Assistant Professor, Pharmacy Systems, Outcomes and Policy, University of Illinois, Chicago

\section{Emily Reeve, PhD}

Dementia Research

Development Fellow, Dalhousie

University, University of

Sydney

Joseph Ross, MD, MHS

Associate Professor of

Medicine, Yale University

James Rudolph, MD

Professor of Medicine,

Brown University 
Gordon Schiff, MD

Associate Director of Brigham

and Women's Center for

Patient Safety Research

and Practice and Associate

Professor of Medicine at

Harvard Medical School

Ian Scott, MD

Director of the Department

of Internal Medicine and

Clinical Epidemiology, Princess

Alexandra Hospital
Nilay Shah, PhD

Health research and policy

consultant, Mayo Clinic

Jay Shaw, PhD

Assistant Professor, University of Toronto

Michael Steinman, MD

Professor of Medicine,

University of California,

San Francisco
Cara Tannenbaum,

MD, MSc

Professor, University of

Montreal; Scientific Director,

Canadian Institutes of Health

Research

Johanna Trimble

Patient Champion, Patients for

Patient Safety Canada
Kristen Zimmerman, PharmD, CGP

Associate Professor, Virginia

Commonwealth University

Andrew Zullo, PharmD,

ScM, PhD

Assistant Professor

Brown University

\section{The following people served on our project's advisory committee:}

\author{
Anthony Barrueta, JD \\ Senior Vice President, \\ Government Relations, Kaiser \\ Permanente
}

John Bulger, DO, MBA

Chief Medical Officer

Geisinger Health Plan

John Devlin, PharmD,
FCCM, FCCP
Professor of Pharmacy,
Northeastern University
Terry Fulmer, PhD, RN,
FAAN
President, The John A. Hartford
Foundation

Leigh Purvis, MPA

Director, Health Services

Research, AARP

Gordon Schiff, MD

Associate Director of Brigham

and Women's Center for

Patient Safety Research

and Practice and Associate

Professor of Medicine at

Harvard Medical School
Michael Steinman, MD

Professor of Medicine,

University of California,

San Francisco

Johanna Trimble

Patient Champion, Patients

for Patient Safety Canada

Jeff Williamson, MD, MHS

Professor of Internal Medicine,

Wake Forest School of Medicine 
